\title{
Designing Biologically-inspired Smart Building Systems: Processes and Guidelines
}

\section{Citation}

Park, Daekwon and Martin Bechthold. 2013. Designing Biologically-Inspired Smart Building Systems: Processes and Guidelines. International Journal of Architectural Computing 11, no.4: 437-464.

\section{Published Version}

doi:10.1260/1478-0771.11.4.437

\section{Permanent link}

http://nrs.harvard.edu/urn-3:HUL.InstRepos:12786003

\section{Terms of Use}

This article was downloaded from Harvard University's DASH repository, and is made available under the terms and conditions applicable to Open Access Policy Articles, as set forth at http:// nrs.harvard.edu/urn-3:HUL.InstRepos:dash.current.terms-of-use\#OAP

\section{Share Your Story}

The Harvard community has made this article openly available.

Please share how this access benefits you. Submit a story.

Accessibility 


\section{Designing Biologically- inspired Smart Building Systems: Processes and Guidelines}

Daekwon Park and Martin Bechthold 


\section{Designing Biologically-inspired Smart Building Systems: Processes and Guidelines}

Daekwon Park and Martin Bechthold

This paper investigates design processes of and guidelines for biologically-inspired smart building systems (BISBS). Within the functional and performance requirements of building systems, biologically-inspired design is explored as the key approach and smart technology as the enabling technology. The Soft Modular Pneumatic System (SMoPS) is developed as a design experiment in order to verify the effectiveness of the BISBS design process. Similarly to how independent cells coordinate with each other to undergo certain tasks in multicellular systems, the SMoPS consists of autonomous modules that collectively achieve assigned functions. Within the soft body of each SMoPS module, sensor, actuation, and control components are integrated which enables the module to kinetically respond to and interact with its environment. The modular design and hierarchical assembly logic contribute to creating a flexible as well as robust building system. Throughout the design process, prototyping, simulation, and animation are utilized as an iterative and diversified development method. 


\section{INTRODUCTION}

Throughout the history of architecture, living organisms have been a major source of inspiration for designers when conceiving a broad range of building elements ranging from ornamental features to structural systems. Architects have pursued this inspiration primarily through an adaptation of geometric and formal similarities, while relying on construction methods and control technologies that "have been within the designer's reach" [I]. This primarily formal approach towards adopting biological precedents has masked the potentially interesting and relevant guidance designers can receive from natural precedents when designing systems to support building operation and controls. This is particularly pertinent in the context of smart buildings with their complex behaviors and interactive features.

The increasing importance of better understanding and promoting the performative aspects of buildings have led to many improvements and advancements in building control systems [2]. Technologies relating to lighting, heating and ventilation, air conditioning, elevators and escalators, and acoustics, to just name a few, have been key areas of development. These individual technologies are increasingly expected to interact as overall systems, especially in the context of smart buildings [3]. As a result, effective control systems needed to be developed in parallel with the building technologies mentioned before, starting from simple pneumatic systems in the 1960s and 1970s to the analogue electronic control devices in the 1980s. With the arrival of digital controls and information technology in the 1990s, the building control system evolved into building automation systems (BAS) which connect the various individual technical systems together using an interlinked network of sensors and actuators. BAS globally monitor and control them within a centralized platform [4].

BAS are now considered integral to smart technology, a field that studies the interaction of dynamic systems in response to the environment. The field of smart technology is inherently multidisciplinary and hierarchical. Smart technology can be hierarchically categorized based on the complexity of the technology and types of application: smart materials; smart elements and control; smart products; smart components; assemblies and systems; and intelligent environments. Smart materials are the basis for many higher level categories, while intelligent environments are assemblies of the lower level categories [6]. Their fundamental attributes include the capabilities to sense, adapt, and respond to the internal or external changes. Key components including a host structure, sensors, actuators, and control elements [5]. These attributes are also the core characteristics of biological systems, a context in which they relate to adaptation and homeostasis. This similarity is one of the key reasons why the field of smart technology often references biological systems during the development process [7].

Smart technology has generally been the domain of scientists and engineers. Recent advancements of digital fabrication technologies and 
processes and the development of innovative material systems and simulation tools are rapidly bridging the technological gap between the field of architecture and the science and engineering disciplines. Other enabling factors for designers to engage with the domain of smart technology are the development of open source data, easily accessible program platforms, and affordable physical computing and prototyping components.

Experimentation in this domain has never been easier. Today, the need to control increasingly complex building systems finally coincides with the opportunities afforded by the democratization of enabling smart technologies. The promise of harnessing nature's principles, of designing and deploying biologically-inspired smart technology building systems (BISBS) is becoming feasible in terms of cost, robustness, and durability.

This paper presents BISBS as novel approach to the challenge of smart building systems. The next chapter defines and characterizes this approach in order to identify the effective guidelines and design processes. In Chapter 3, Soft Modular Pneumatic System (SMoPS) will be presented as a design experiment for testing the BISBS design process and guidelines. Finally, Section 4 will evaluate the SMoPS development process and design guideline in the context of BISBS.

\section{BIOLOGICALLY-INSPIRED SMART BUILDING SYSTEM}

Biologically-inspired smart building systems (BISBS) are integrated building systems that incorporate concepts and principles of biologically-inspired design using the advances in smart technology as the enabling technology. As seen in Figure I, the intersection of biologically-inspired design, smart technology, and building systems is at the core of BISBS (Figure I).

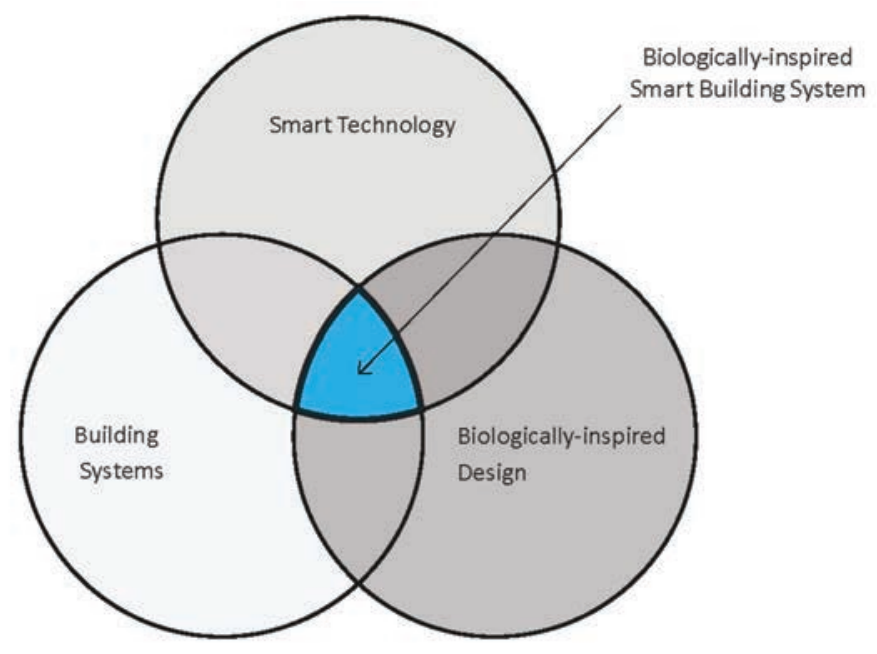

Figure I: Biologically-inspired Smart Building System - Venn Diagram. 
Figure 2: Other intersections of the Biologically-inspired Smart Building System: (a) Biologically-inspired building systems design; (b) Responsive and/or interactive Installation, art, or products; (c) Dynamically controlled building systems.
If we only consider the intersection between building systems and biologically-inspired design, the advantages and opportunities of smart technology are not utilized (Figure 2, (a)). Although many noticeable buildings were designed using biological inspirations, much of this work focused on the aesthetics of the natural forms. Advancements materials, construction and structural analysis enabled architects and engineers including Buckminster Fuller and Frei Otto to successfully integrate biological inspirations with structural design. With the advancement of smart technology, architects and engineers can now experiment and integrate interactive and dynamic capabilities into a variety of building systems.

On the other hand, the intersection between smart technology and biologically-inspired design alone would merely fall in the domain of responsive and/or interactive installation or art, excluding the possibility for building applications (Figure 2, (b)). The sculptures and installations of Philip Beesley [8], for example, successfully combine biologically-inspired design approaches with smart technology to create unique spatial experiences, but are difficult to be use as building systems due the fragility of the materials and the complexity of the assembly.

Finally, the intersection between smart technology and building systems cannot take advantage of the possibilities that the biologically-inspired design approach can offer (Figure 2, (c)). BAS is a good example of utilizing sophisticated smart technologies to monitor, regulate, and control building systems. However, since BAS are generally concealed from the public and limited to the operation and maintenance of building service systems, it tends to lack the qualitative and experiential potentials that biologicallyinspired design can provide.

BISBS organizes technical infrastructure in terms of system layout and control principles, allowing multiple architectural systems to interface efficiently. Its core advantage is the achievement of flexibility and robustness by adopting modularity and hierarchical design principles found in biological systems. The overall organization and developmental process of BISBS is summarized in figure 3.
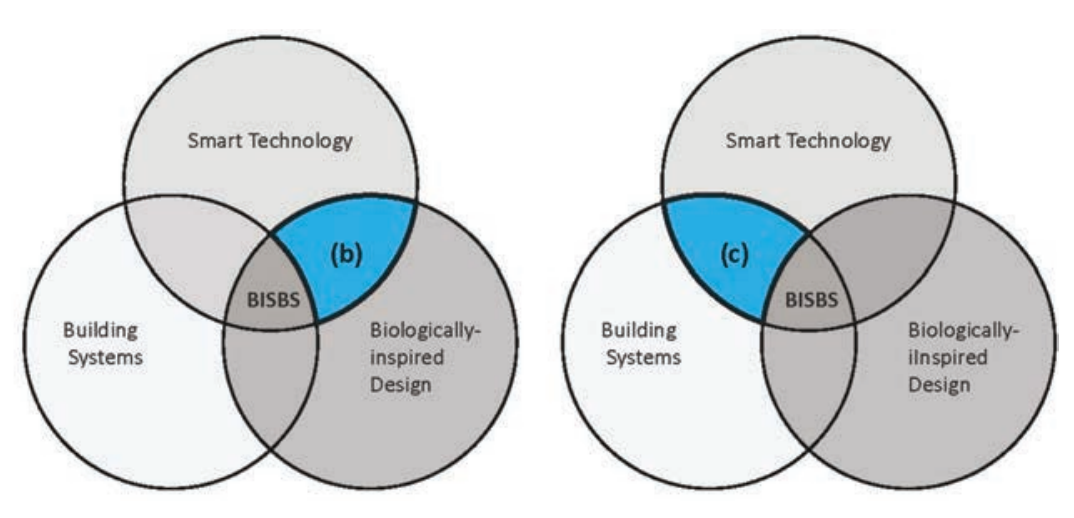

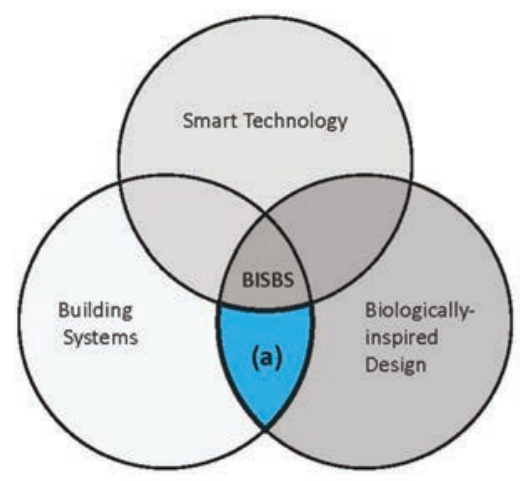




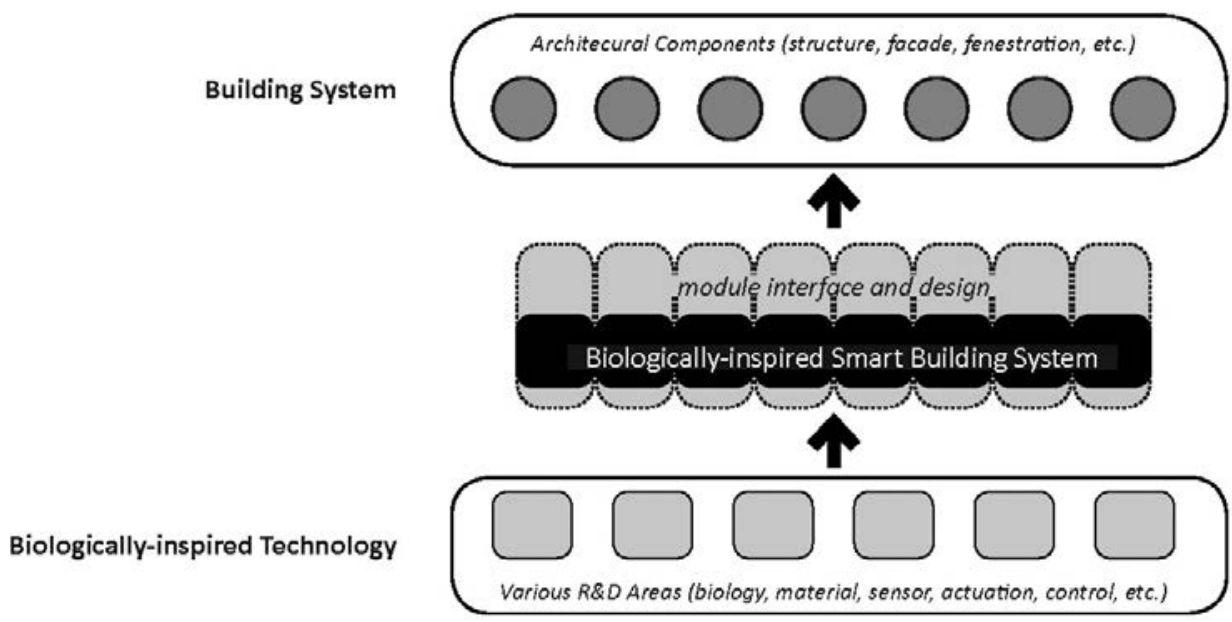

Figure 3: Biologicallyinspired smart building system diagram.

The key characteristics of BISBS are summarized below. These serve as guidelines during the development process:

- $\quad$ BISBS actively refers to biological systems, especially to physiological processes, in order to effectively generate and develop new ideas. Its advantages include modularity, robustness, kinetic proofreading, and hierarchical design.

- $\quad$ BISBS relies on multidisciplinary research methods during the development process. It integrates various developments in material science, computer science, mechanical engineering, electrical engineering, biology, etc. as the building blocks to combine into a complex system of active building elements and their controls.

- $\quad$ BISBS is an active system that dynamically responds to and interacts with the environment and people. The key components of smart technology (e.g., sensor, actuation, and control) are the enabling technologies to achieve this.

- In order to meet the rigorous requirements of an architectural system, significant emphasis must be given to deploying means and methods of efficient fabrication and material assembly that can achieve flexibility, robustness, durability.

- $\quad$ BISBS is a flexible and versatile modular system that becomes the technology infrastructure for other building systems (i.e. envelope, services, structural, interior, and site [9]). It is the entity that bridges these systems together. 
Figure 4: Pneumatic system and component layout of SMoPS.

\section{Design Experiment: Soft Modular Pneumatic System}

\section{I. System overview}

In order to develop the BISBS design process in detail, as well as to validate its effectiveness, a design experiment was developed that is representative of the challenges present in designing complex, interactive building systems. Soft Modular Pneumatic System (SMoPS) is a dynamic pneumatic interface which can be used in building applications including responsive façade, ceiling, floor and interior screen, etc. Among the various strategies found in biological systems, SMoPS explores modularity, homeostasis, and responsiveness to stimuli. Similar to how independent cells coordinate with each other to complete certain tasks in multicellular systems in nature, the SMoPS consists of autonomous modules that collectively achieve assigned functions. Within each SMoPS module, sensor, actuation, and control components are integrated in a soft body, contributing to the robustness of the system as well as allowing it to adapt to external stimuli. As an aggregated system, SMoPS has the potential to initiate emergent behaviors at the global level due to the chain reactions between individual modules at the local level.

SMoPS' pneumatic system works similar to the cardiovascular system in humans. Biological cardiovascular systems circulate blood from the heart to the rest of the body and vice versa in two closed loop systems (pulmonary circulation and systemic circulation) in order to maintain homeostasis. Similarly, the pneumatic system of the SMoPS maintains two loop systems: a negatively pressurized loop and a positively pressurized loop, which are connected to external pneumatic pumps (Figure 4).
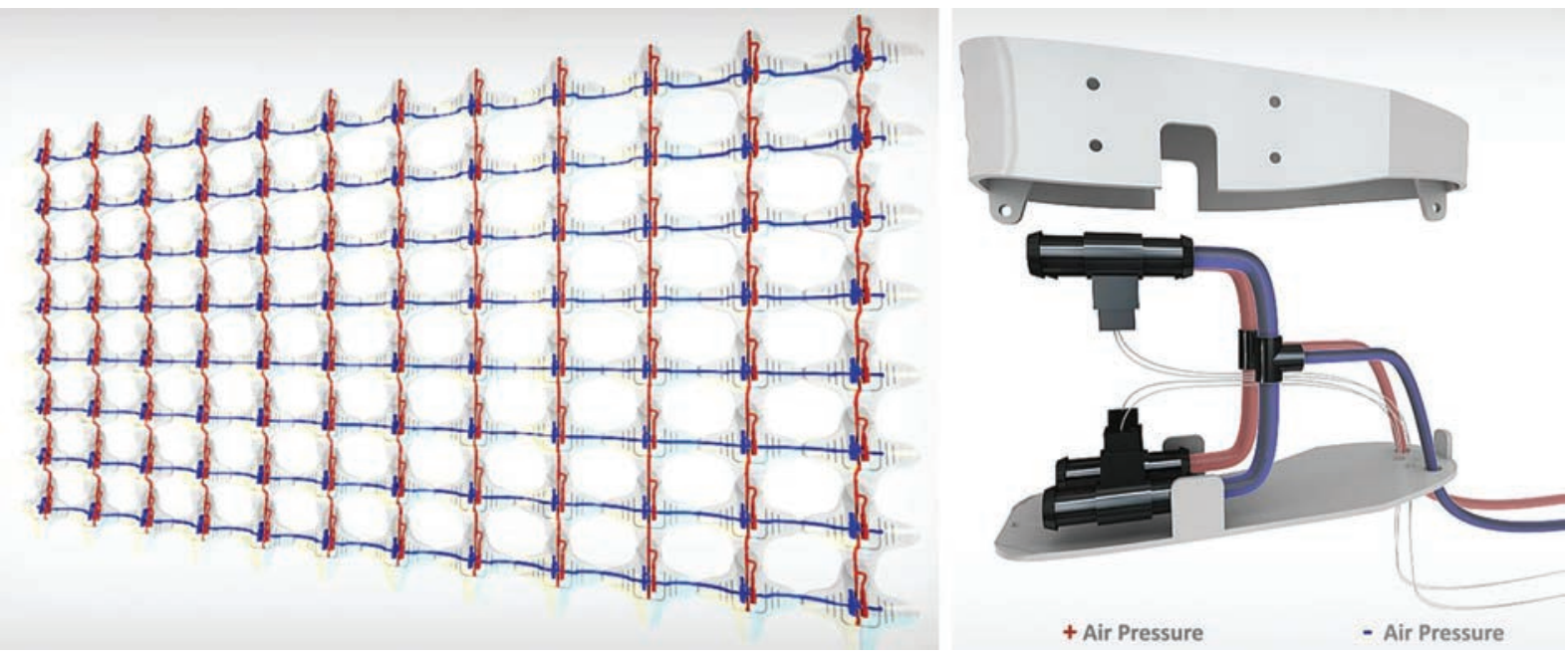

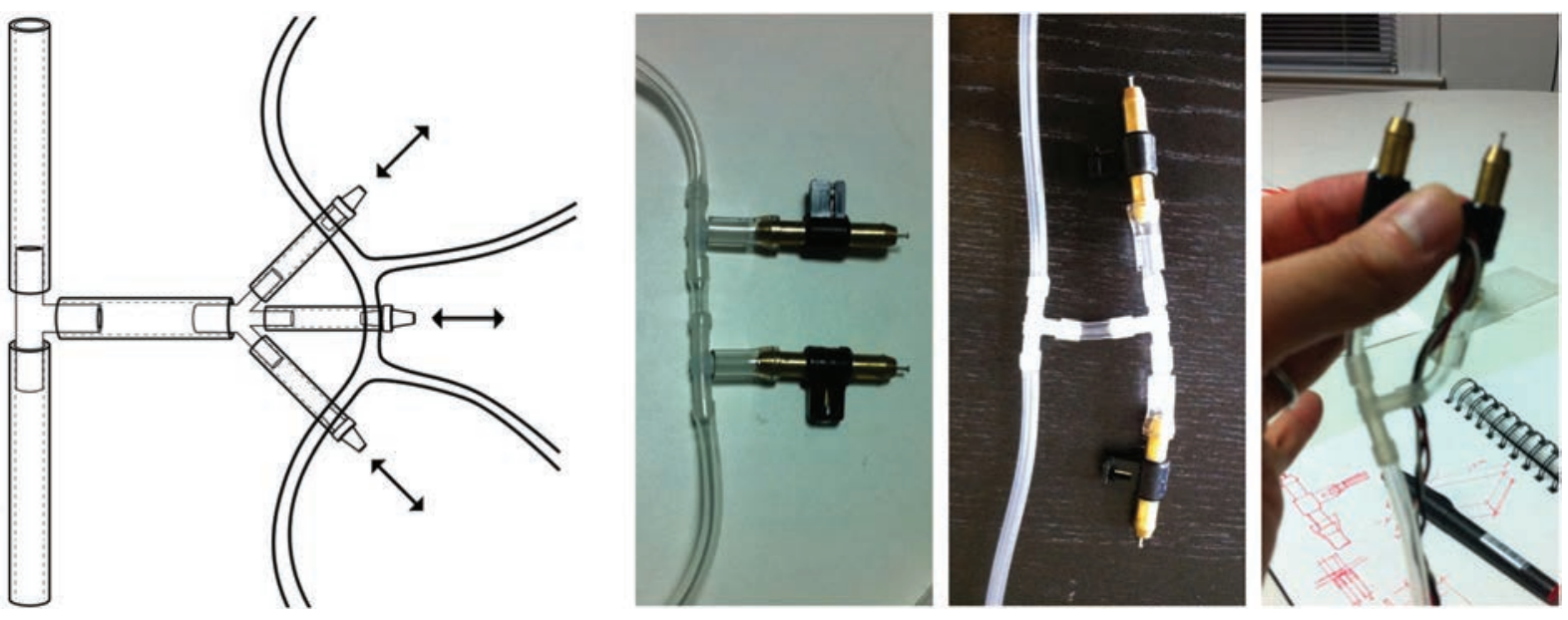

A Figure 5: Independently controllable

From the two closed loop system, any number of independently controllable valves can be attached hierarchically like the branch development in trees or in vascular system (Figure 5, (a)). In order for this pneumatic system to operate, the two closed loops must constantly maintain a target pressure (one being negative and the other being positive), responding to the valves opening and closing. This pressurized air is the power source that operates all actuators.

There are two main feedback loops working in the overall circuits of SMoPS. Within the module itself, the feedback system consists of the embedded sensors, soft body actuators, microcontrollers, and valves. In this loop, the data from the sensors are sent to the microcontroller to be processed, and the microcontroller sends the output signals to open or close the valves to allow pressurized air to go into or out of the soft body (Figure 7). The geometry of the soft body can deform based on how the air circulates internally. The second feedback loop, which can be externally attached to the systems, consists of a pneumatic pressure sensor, a microcontroller, and a pneumatic pump. The microcontroller constantly receives the pressure value from the pneumatic pressure sensor and regulates the pneumatic pump to achieve the target pressure. If the first loop is analogous to the living organism's responsiveness to stimuli, the second loop can be compared to an organism's maintenance of homeostasis (Figure 6).

The key advantage of this dual-feedback loop system is that it only requires two pumps to power and regulate the entire system and, simultaneously, achieve independent local-level control. In addition, there can be two distinctive methods of control, distributed and centralized, due to the internal and external microcontrollers. When there is a failure in the external loop, it can be isolated and fixed either by modifying the components or simply replacing them, which is possible because there is valve: (a) Branch configuration of the valve; (b) perpendicular configuration; (c) parallel configuration; (d) $y$ connection configuration. 
Figure 6: Dual-feedback loop of SMoPS.

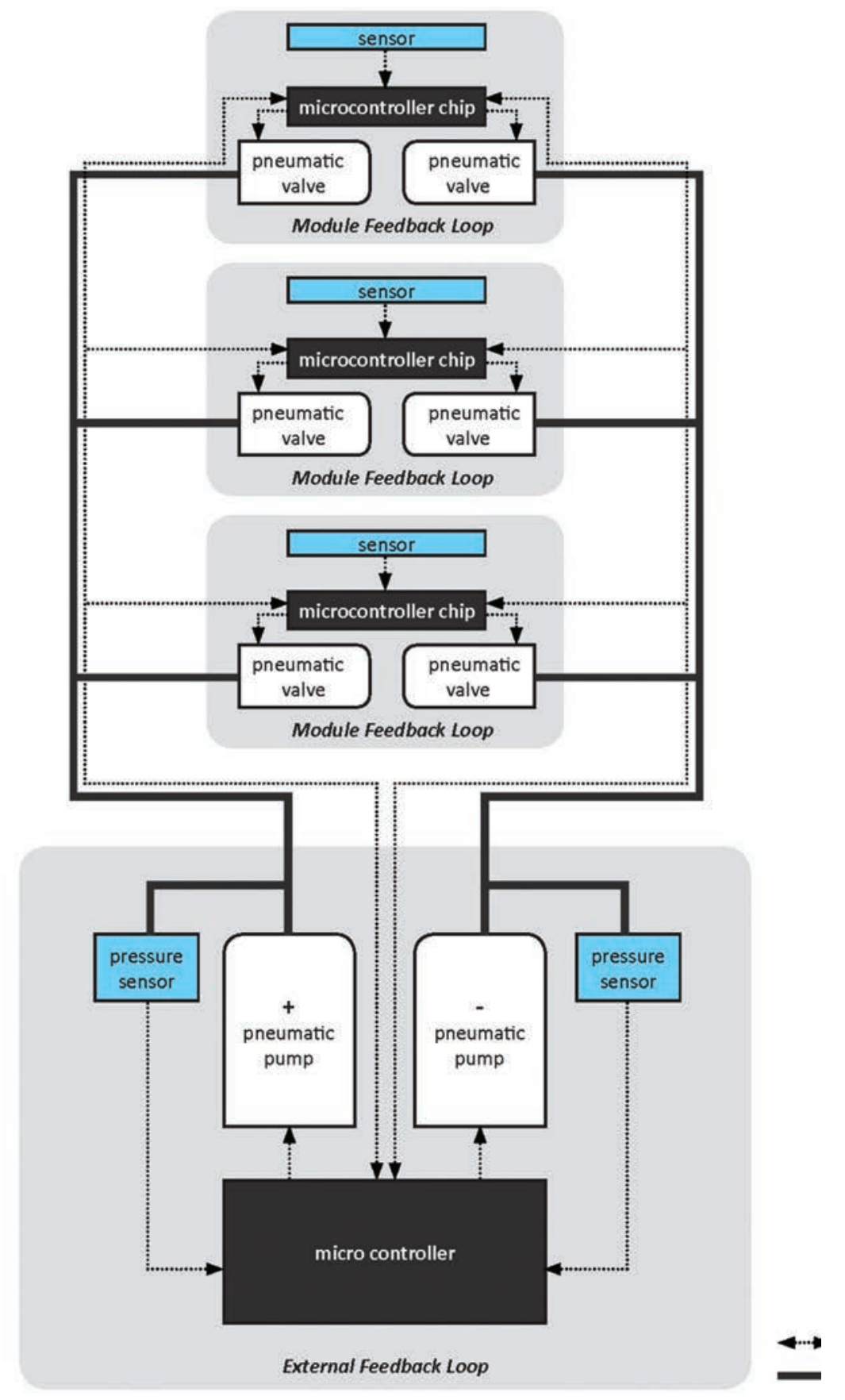

one source of power connected to the entire system. On the other hand, when one or several modules fail (each having its own second loop), the overall system still functions as long as the modules are connected as a closed network configuration. The modules can be taken out, fixed, and inserted back into the system any time. 


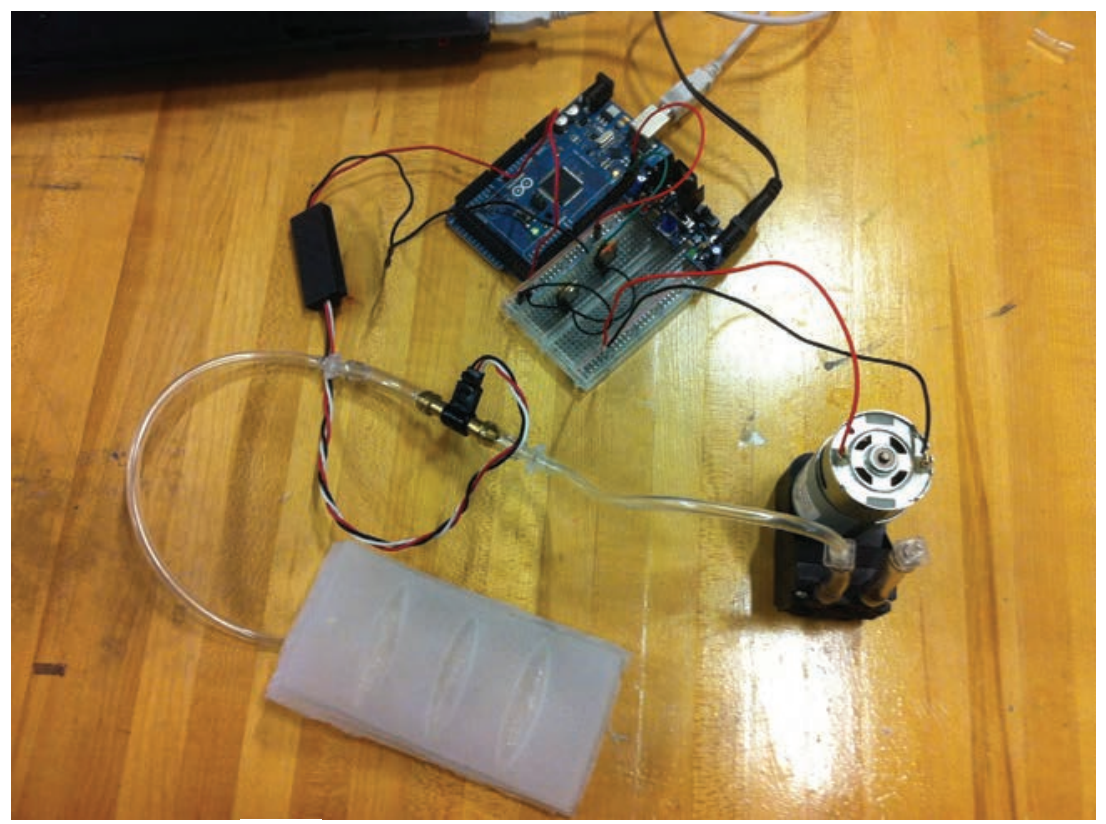

Figure 7:Valve, pneumatic pump, and microcontroller connection prototype.

\subsection{Actuator, sensor, and control}

The kinematics of SMoPS are based on the combination of an elastic material body actuator and a pneumatic pressure feedback system, inspired by the muscular-hydrostats in nature (e.g., elephant trunk, starfish, octopus arm, etc.) [10]. The actuation system is based on George Whiteside's research on soft robotics using the geometry of the channels embedded in the elastomers [ I I]. The key principle of deformation (actuation) is the varying elasticity of the channels (Figure 8 ), achieved either by varying the section thickness with same material or by using the same section thickness with materials that have varying degrees of elasticity. By strategically utilizing asymmetric deformation during the inflation in a network of channel systems, one can control the overall deformation of each module.

In the study of the module's kinematic modes three types of motions are categorized: bending, expansion, and extension (Figure 8). The bending movement is achieved by subdividing the air chamber into channels that run perpendicularly to the direction of deformation. The denser the channels, the tighter the bending radius will be, due to the appended effects at the overall deformation. The expansion and extension motions work similar to a balloon. In addition to the overall shape of the unit, embedding redundant geometries by folding or convoluting material allows further extension to the overall deformation (Figure 8). 
Figure 8:

Kinematic types and properties.

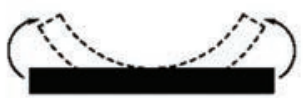

Bend

multiple channels volumn subdivision
Default mode

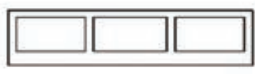

section thickness variation

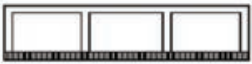

material differentiation
Active mode

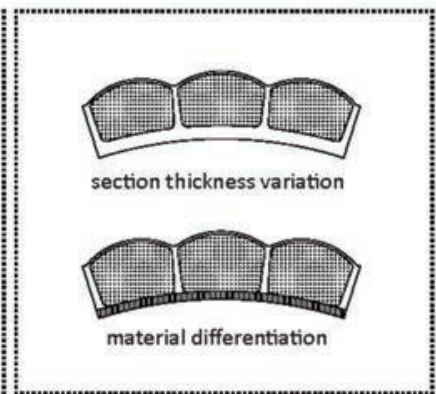

Active mode

Default mode
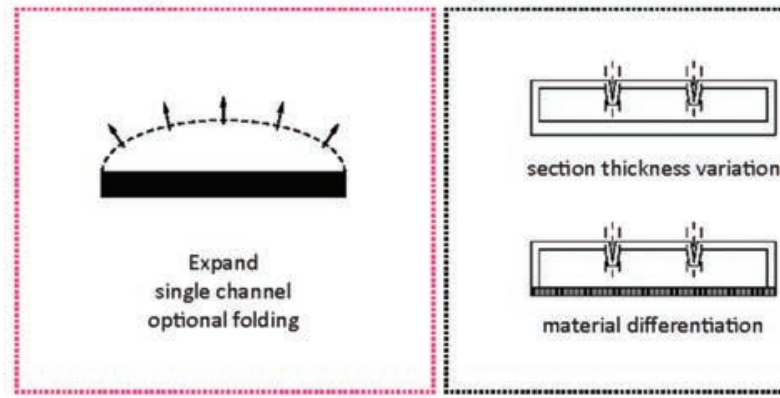

section thickness variation

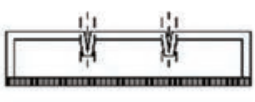

material differentiation

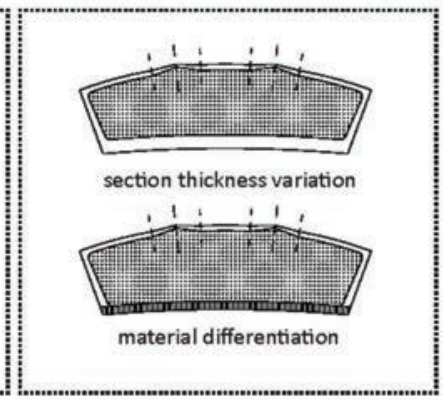

Active mode

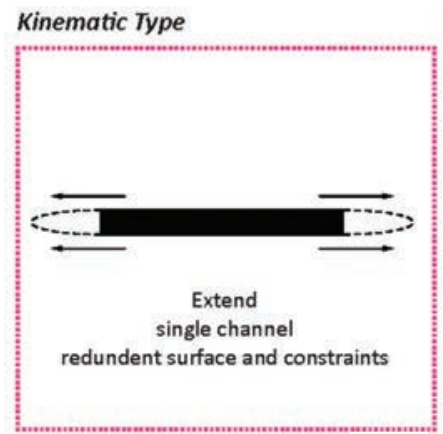

Default mode

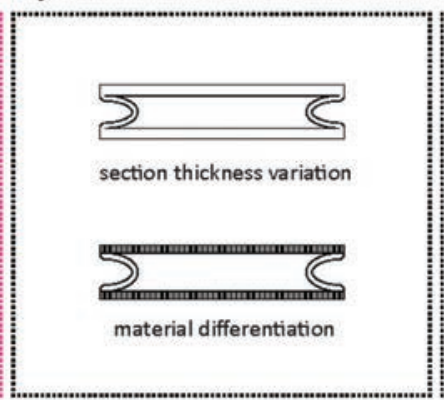

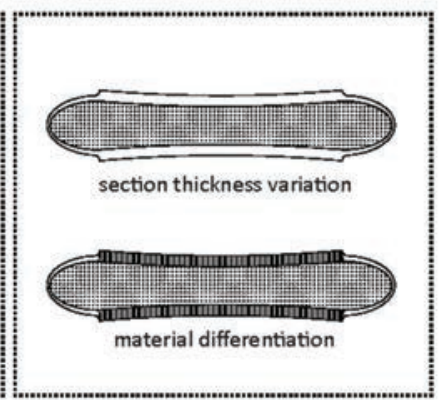

SMoPS utilizes a capacitive sensor technology which can measure the dynamically-formed capacitance created between a uniformly charged conductive layer and a conductive object, separated by a dielectric material (e.g. air). This technology allows the capacitive sensor to measure the presence as well as proximity of conductive objects like human hands [I2]. The main strategy for making the body of the module conductive is to inject conductive gel, commonly used for heart rate monitoring, directly into the hollow channel pattern created within the soft body itself. By utilizing a gel-type conductive material, it is possible to maintain the softness of the SMoPS body (Figure 9). 


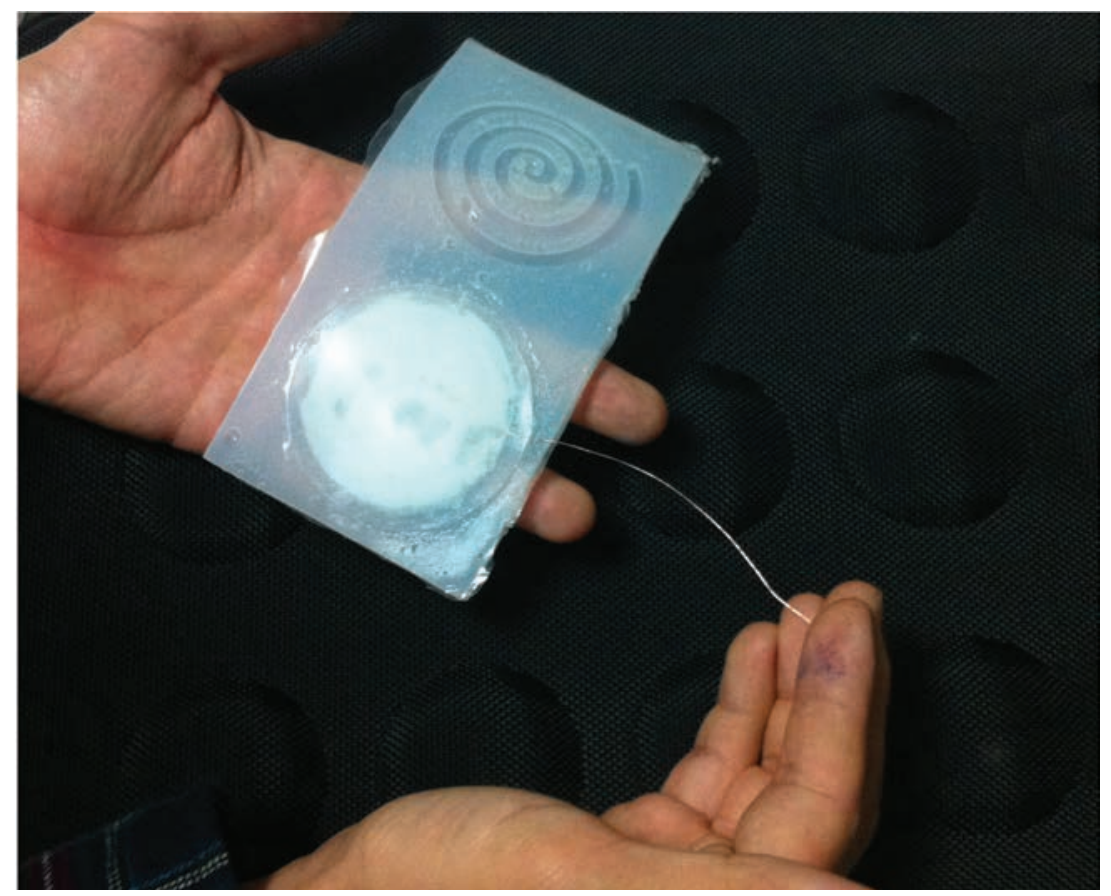

SMoPS modules can form a capacitive sensor grid that can measure the specific locations where proximity and presence of people is detected within the overall field. In this configuration, each SMoPS module functions as a node of the capacitive sensor grid. By measuring the capacitance value at each node and comparing the relative difference between the transmitter value and the receiver value, the SMoPS field can function as an interactive surface that detects proximity or touch (Figure I0).
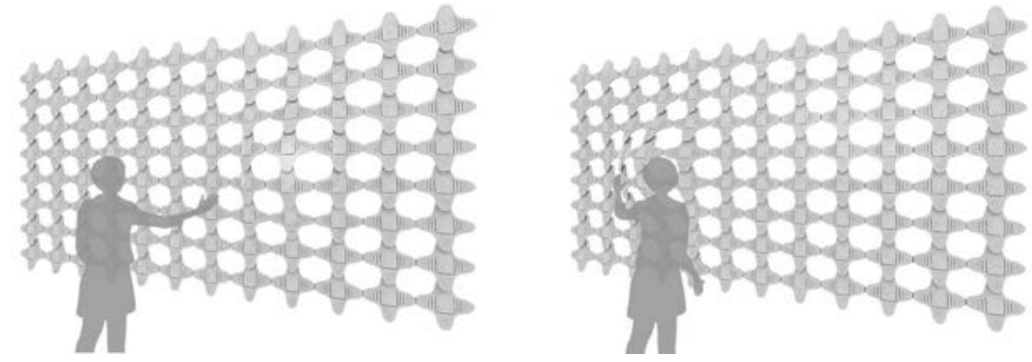

The assembly of the SMoPS modules utilizes two different methods. The vertical connection of the modules is through a simple hanging mechanism using the connection rings embedded in each module. The horizontal connection is achieved through luer lock connectors. Since the vertical
Figure 9: Embedded capacitive sensor prototype.

Figure 10: Responsive surface.

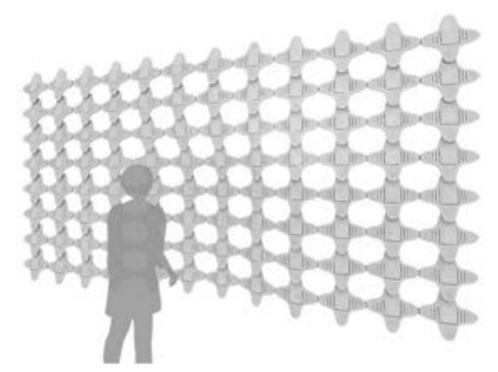




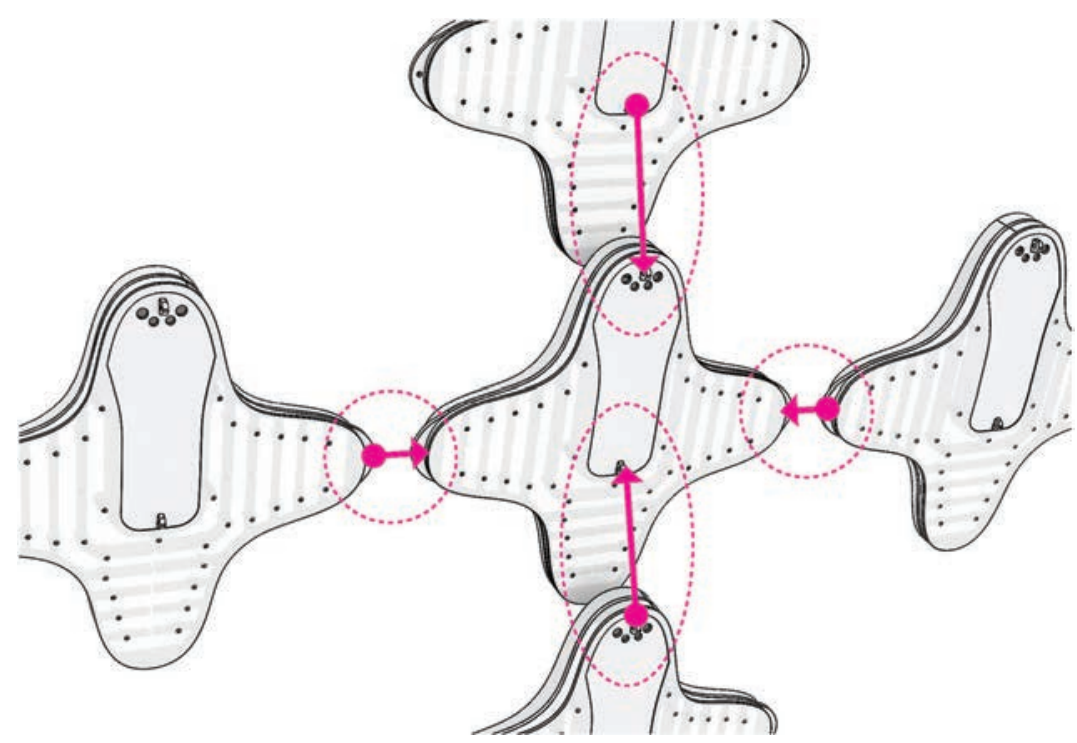

connection is located in the center of the module, which is the static part, the actuation of the module does not affect the top or bottom modules. On the other hand, due to the horizontal linkage, the actuation of the module affects the adjacent modules horizontally (Figure II).

The aggregated system can be controlled by using two distinctive methods: a central control method and a distributed control method. The central control method utilizes the external microcontroller as the master and the microcontroller chips that are embedded in each module as the slaves. When the modules are assembled to a field, each slave microcontroller chips is registered to the master microcontroller. This control method allows the master controller to control each slave microcontroller in order to perform specific tasks (e.g., information display, interaction, etc.). The tasks can be controlled globally and the patterns or sequence of movements can be predefined, or they can be generated using algorithms (such as cellular automata, flocking, etc.) (Figure 12, left).

The second method of control is the distributed control. In this setting, the microcontroller chips that are embedded in the modules are utilized as independent agents. When a sensor of a unit or a group of units is triggered, the actuations (responses) occur based on the program logic of the agents (embedded microcontroller chip). These state changes, including movement and deformation in the active module(s), physically affect the adjacent modules and create chain reactions that initiates emergent behaviors at the global level. (Figure 12, right)

The testing of the aggregation of the modules was conducted using a combination of virtual simulation and physical methods. Virtual methods consisted of a parametric 3D model constructed using Rhinoceros and Grasshopper, while the physical methods consisted of a system of 

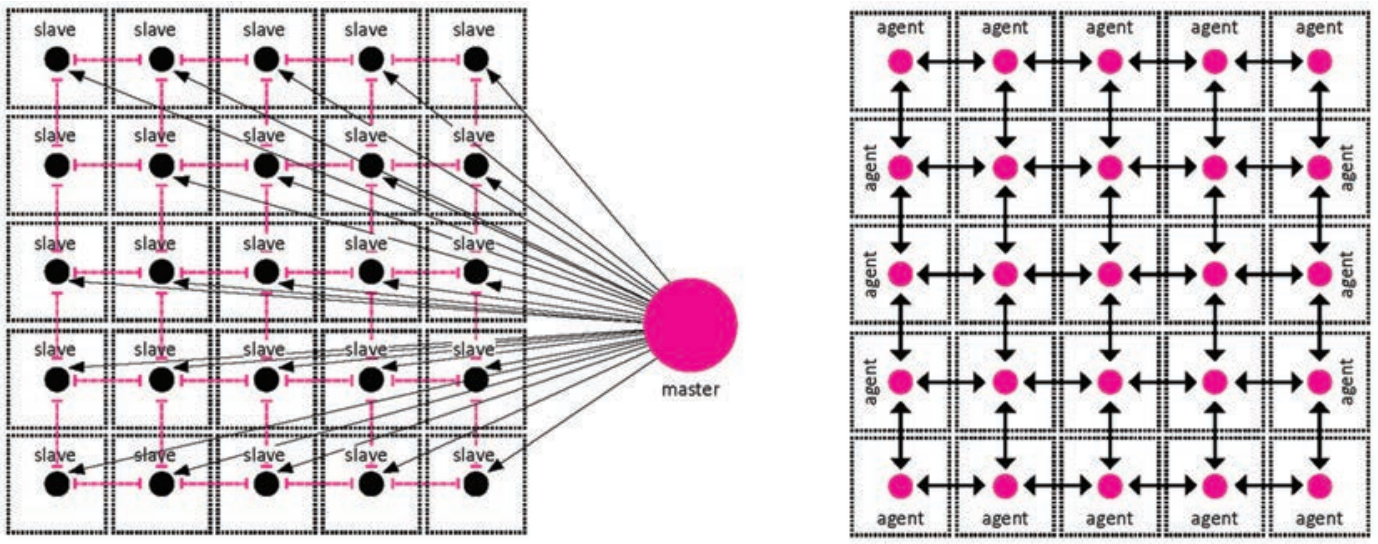

Figure 12: Control logic. Left: Centralized control. Right: Distributed control.

microcontrollers and sensors. The main platform for bridging between the virtual and the physical methods is the Firefly plugin for Grasshopper [13].

V Figure 13: Simulation using physical sensors to manipulate digital model.
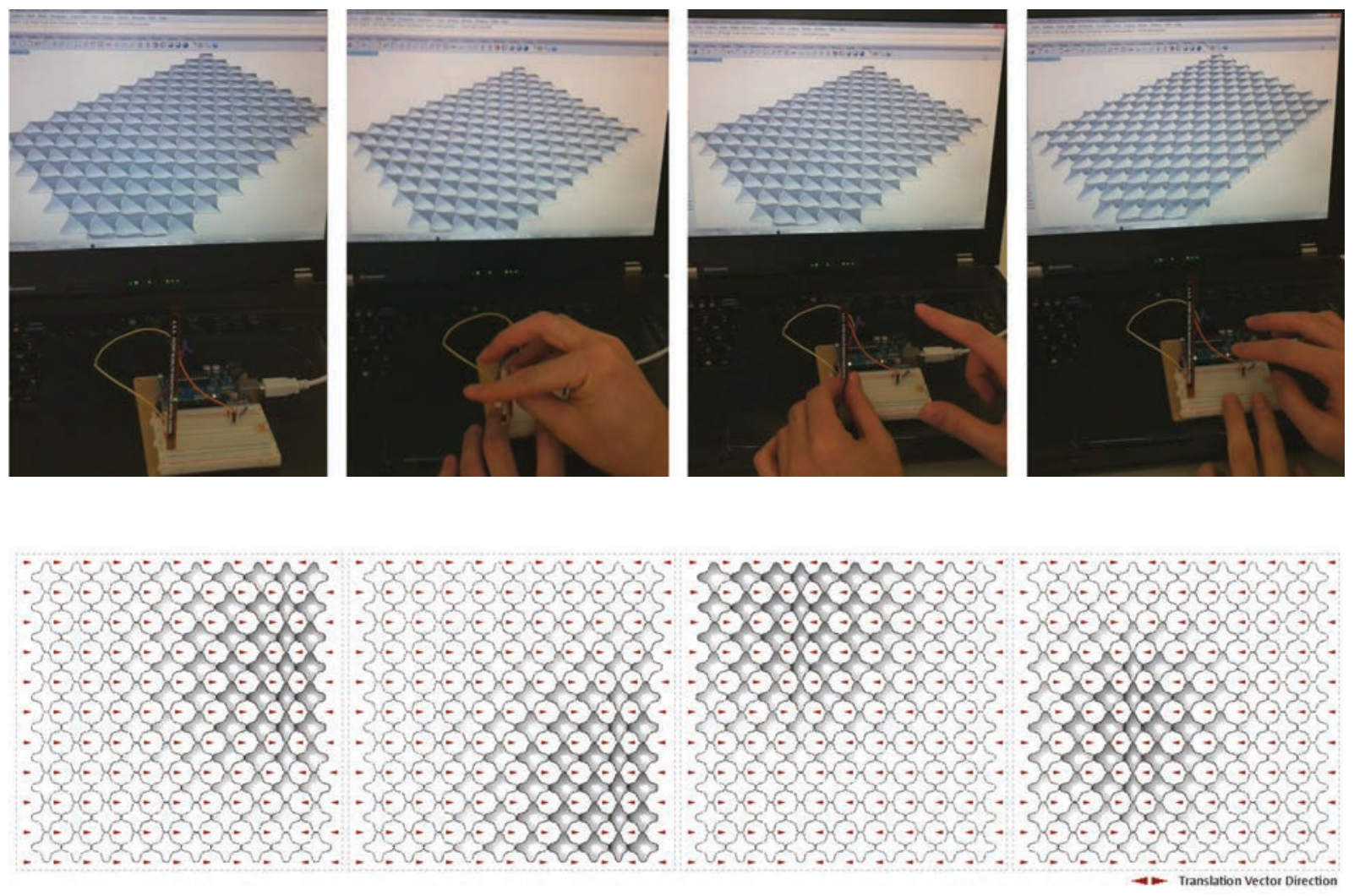

$\Delta$ Figure 14: Field differentiation. 
$\nabla$ Figure I5: First stage mold and cast geometry and prototype.

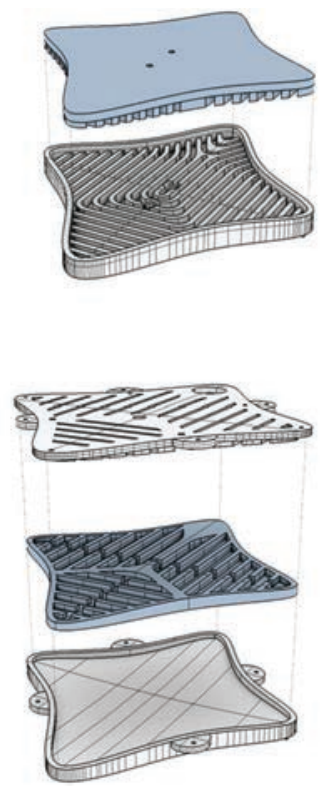

\subsection{Prototype design and fabrication process}

Prototyping during the development of the SMoPS went through three iterative stages, each having a specific objective that informed the following stages. The first stage of prototyping focused on materiality, investigating the overall fabrication methods, soft material properties, and the geometry of the air chambers and channels. A three-part mold was created using a 3D printer (Eden Polyjet 500v) and the soft module shells were cast with the silicon based elastomer (Ecoflex 050). The top layer consisted of a flat sheet with deep grooves engraved in it, and the bottom layer consisted of two separated air chambers and channels. The thickness of the cast layer is significantly larger in the top layer than in the bottom layer, thus controlling the direction of the deformation (Figure 8).

The grooves of the top layer were intended to guide the deformation, and the channels along the bottom layer have a continuous gap running perpendicularly through the channels to allow air to flow throughout the air chambers. Once the top and bottom layers are cast, they are glued together using a silicone based adhesive (Sil-Poxy).

Initial actuation/deformation tests pressurized opposite air chambers used a syringe (Figure I5). The channel geometry that creates the bending motion was tested in two separate air chambers ( $A$ and $B$ ). Chamber $A$ exhibits a flapping motion when inflated and deflated (Figure 16, left); and chamber $B$ bend upwards when inflated and downwards when deflated (Figure 16, right).
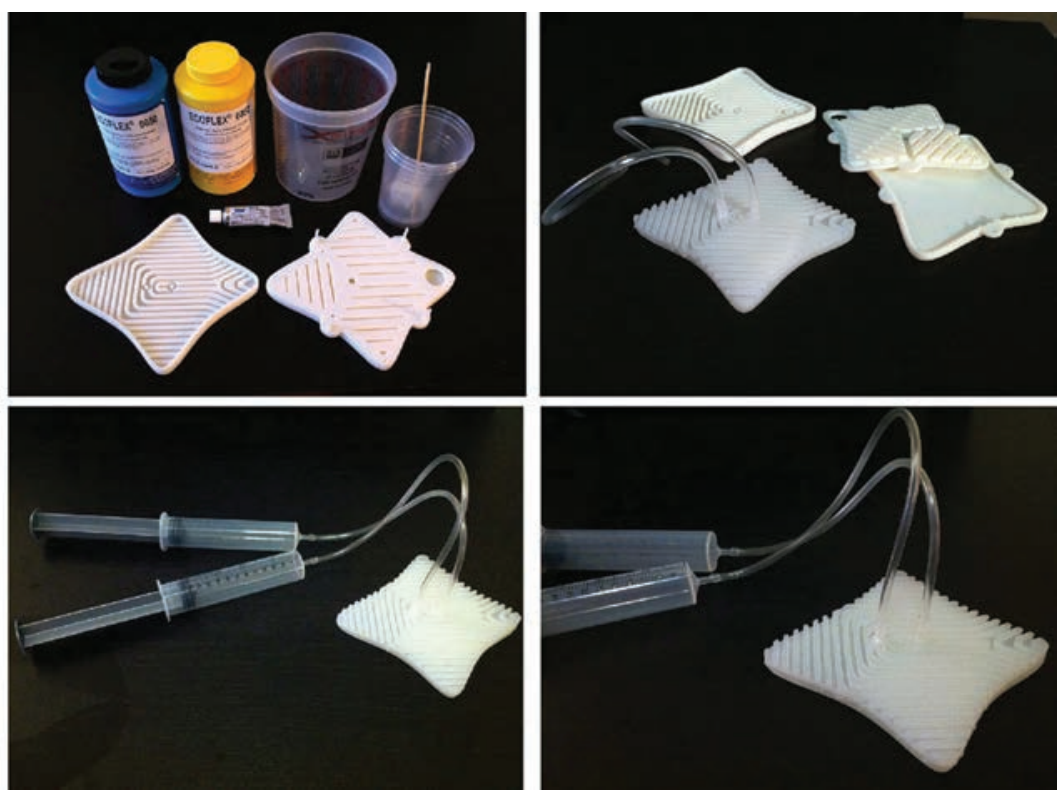

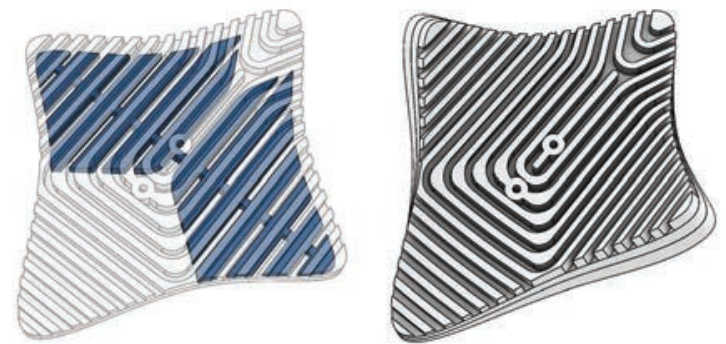

The outcome of this test revealed several problems that needed to be addressed in the second stage. The continuous geometry of top layer's deep grooves hindered the movement rather than guides the movement of the modules. The difference in elasticity (achieved by a different thickness) between the top and bottom layers was too severe for effective movement. In addition, the adhesive seal between the top and bottom layers detached internally (inside the air channels) during inflation. The adhesive bond was too weak to withstand the air pressure - a significant problem considering the challenge of repairing these areas without completely detaching the top and bottom layers (Figure 17).
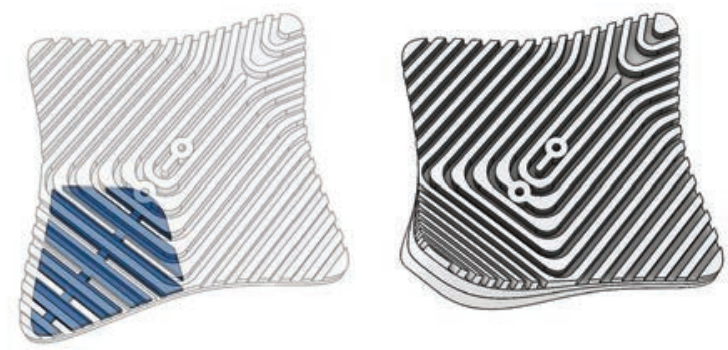

$\Delta$ Figure 16: Chamber location and deformation. Left: Chamber A. Right: Chamber B.
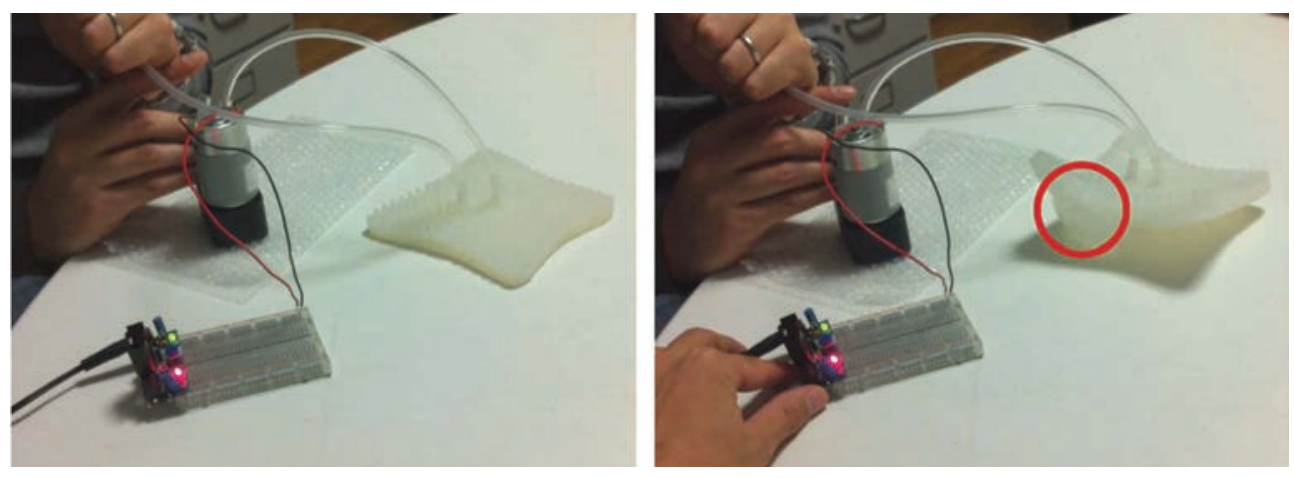

Figure 17:Air channel detachment issue during actuation.

The second stage of prototyping focused on understanding three key issues: first, to better understand the relationship between the module geometry and the type of motion it produced; second, to achieve module deformation by using materials with different elasticity on opposite layers instead of varying the cross-section with identical materials; and finally, to test an external connection element in an attempt to solve the issue of the seal detachment within the air chambers. Base on the kinematic types, three separate units were fabricated and tested (Figure 18).

The 'bend' unit in this phase has a series of air channels to guide the motion. The external connection element works like a clamp, with two strips of plastic bands that are connected together with plastic nuts and 
Figure 18: Second stage mold and cast geometry and casting process
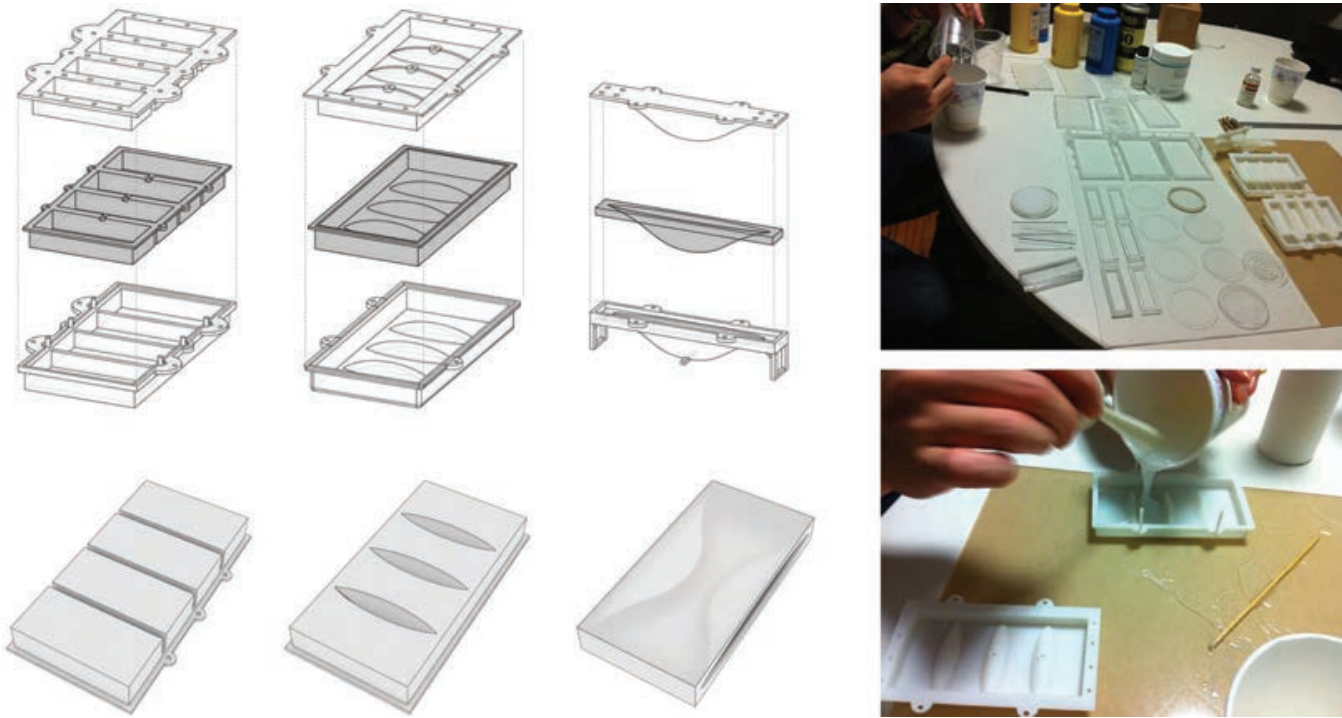

bolts. The 'expand' unit has no channels, so when air pressure is applied it expands in all directions. An indentation pattern is introduced to the surface of this unit to experiment with the possibility of pattern morphing. The 'extend' unit works similarly to the 'expand' unit, but in a more controlled manner. The area that is designed to extend has a redundant surface that is initially folded inside the soft body.

In addition to the kinematic-type study, experiments using different materials were carried out in order to understand whether a wider range

Figure 19:Actuation test.
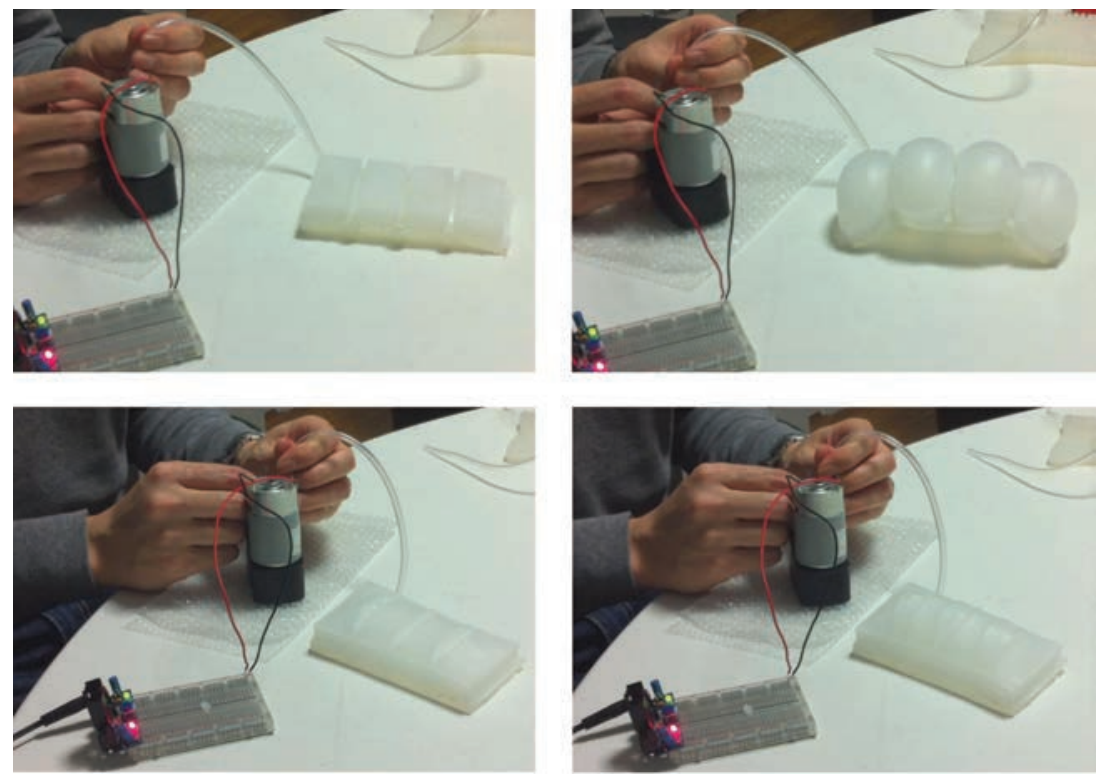
of motion can be produced by than was the case when varying the thickness while keeping material elasticity constant. For this experiment, Polydimethylsiloxane (PDMS) and Ecoflex 050 and Ecoflex 030 are tested (here sorted from smallest to largest shore value or stiffness). Unlike in the first stage of prototyping, the section thickness was kept constant throughout the units.

This phase of testing revealed that varying the materials made the fabrication process more complicated, without yielding significant advantages in performance. Additional problems were revealed during the testing of the external connection element. When the side surface was eliminated in order to accommodate the external clamping element, the bending motion diminished significantly.

The third prototyping stage intended to bring together the previous iterations as well as the pneumatic systems and control elements. The goal for this stage was to scale up the prototype module to the scale of an architectural façade, and embed the sensor, actuation, and control elements into it. The connection method for aggregation was now fully implemented, and the actual tube connectors were used. A hard chassis system was introduced into the design to allow for structural reinforcement, as well as to house the technical components including tubes, valves, and the microcontroller. Furthermore, a modified version of an external clamping component, which conserves the side geometry of the air channel, was designed and implemented (Figure 20).

Two air chambers were designed into the system in order to test the combination of the three kinematic types. The top layer was a combination of extension and expansion kinematic types, having one air chamber (without the channels) that stretches across the top surface, and embedding a deep, folded groove onto it. When the upper layer of the module is activated, it expands like a balloon and further extends in area with the folded geometry. The bottom layer utilizes the bend kinematics with a series of connected air channels that run perpendicularly to the direction of bending. When a positive pressure valve is activated, the unit bends toward the top and vice versa when the negative pressure valve is activated. By varying the pressures within the two chambers in different combinations, a wide range of kinematic states can be achieved (Figure $2 \mathrm{I}$ and Figure 22).

Between the top and bottom layers, another soft sheet of the same material (Eco flex 050) is inserted. This not only functions as the partition for the two air chambers, but also as a structural element that enables the skin to attach to the chassis. The actuator is designed to embrace the entire surface of the chassis except for the back side which can be opened for accessing the technical components. Silicon-based epoxy and the external clamping component are used to assemble the soft body, and mechanical connections (nuts and bolts) are used to attach the soft body to the chassis.

The chassis was fabricated using the 3D printer (Eden Polymer 500v). It 


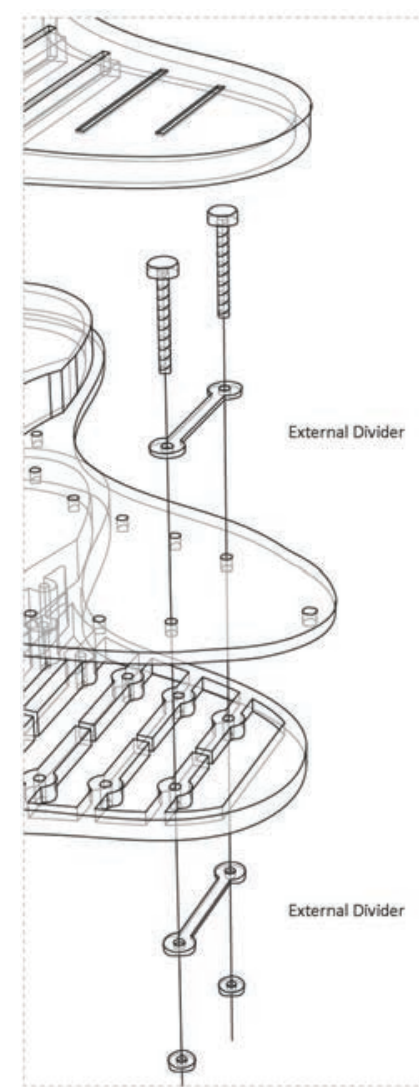

A Figure 20:Third stage mold and cast geometry and prototyping.
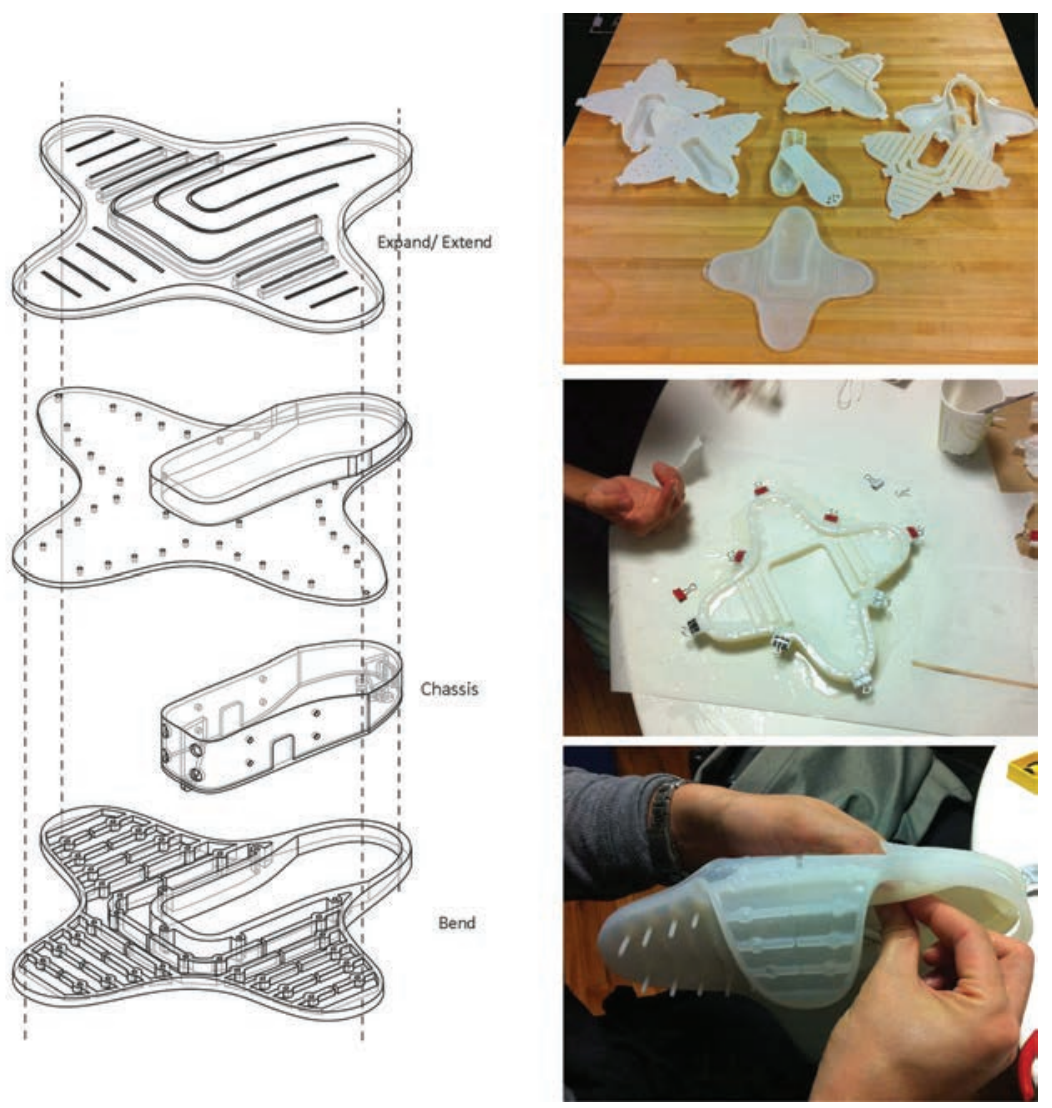

consists of the body and a lid. A microcontroller chip and the y-connection valves (Figure 5, (d)) connected to the tubes are inserted into the chassis. One pair of valves is connected to the positive pressure tube, while the other pair is connected with the negative pressure tube. Depending on the control loop (sensor, actuation, valve control) settings, each valve will open and close to execute the intended task (Figure 22). Two rings that are also integrated within the body of the chassis function as connectors for the aggregation of the modules.
A Figure 21: Left: Bend test. Right: Expansion and extension tests.
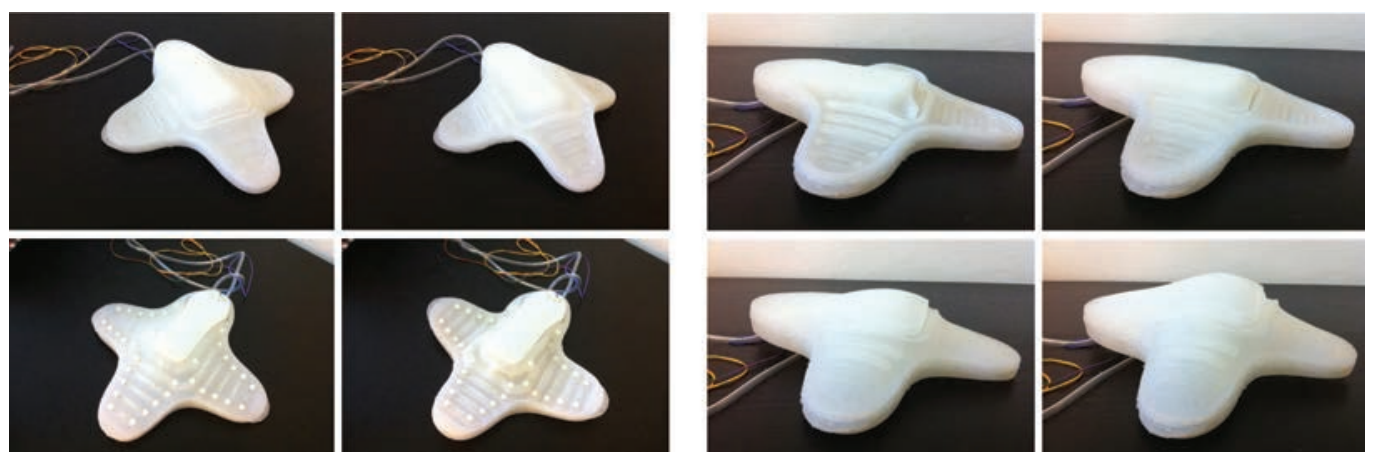

Designing Biologically-inspired Smart Building Systems: Processes and Guidelines 

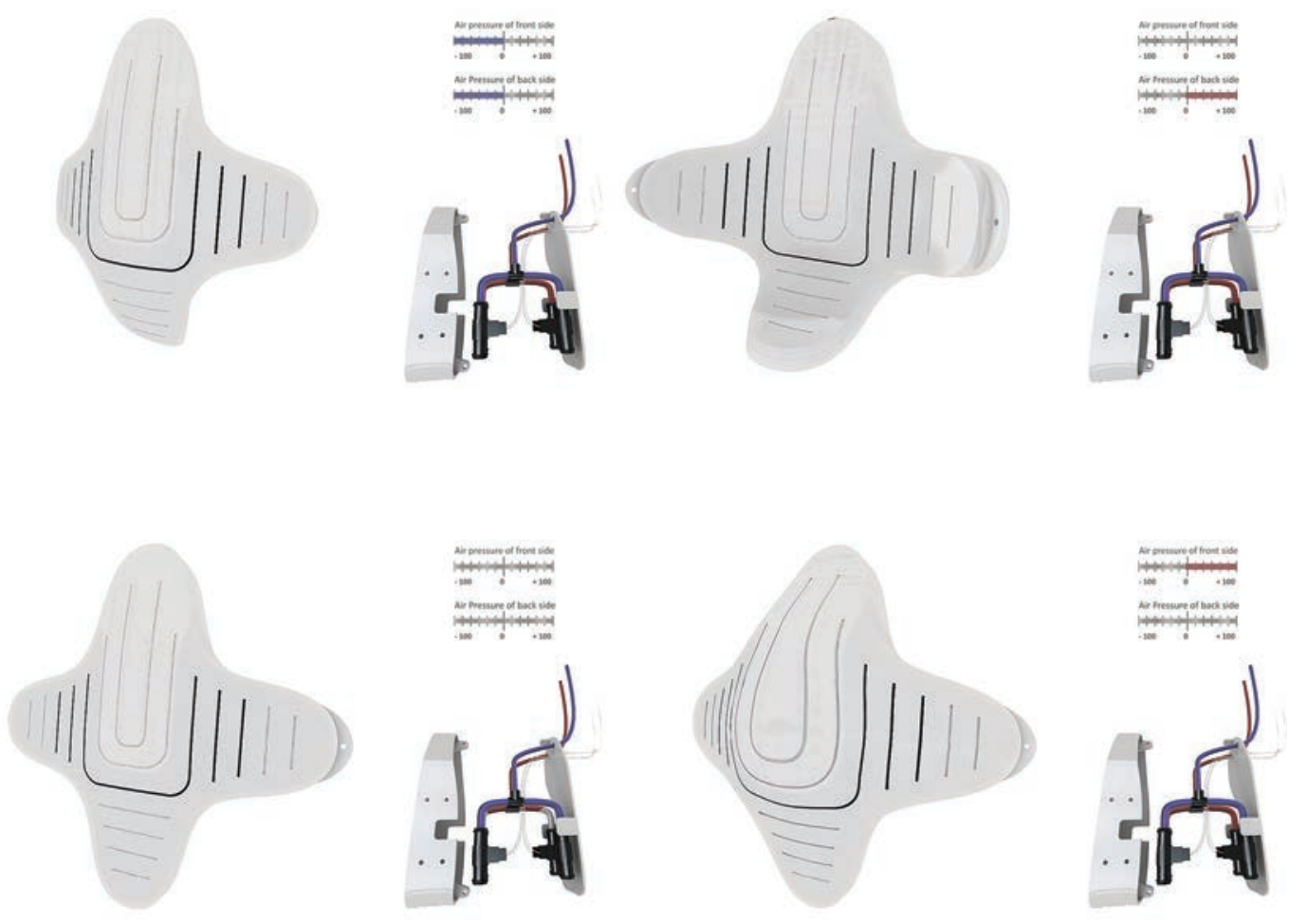

Ar nunum of bath vice
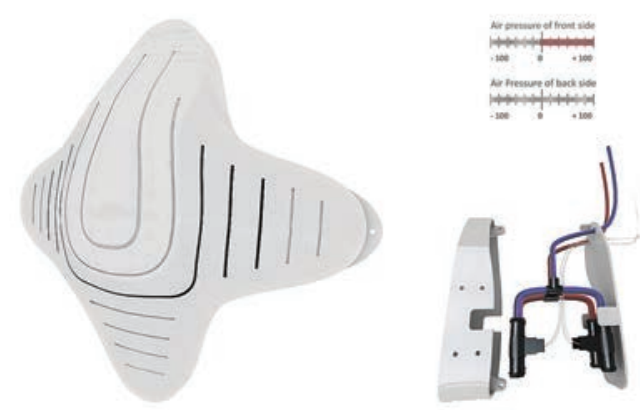

Figure 22:

Actuation logic during third

stage

prototype.
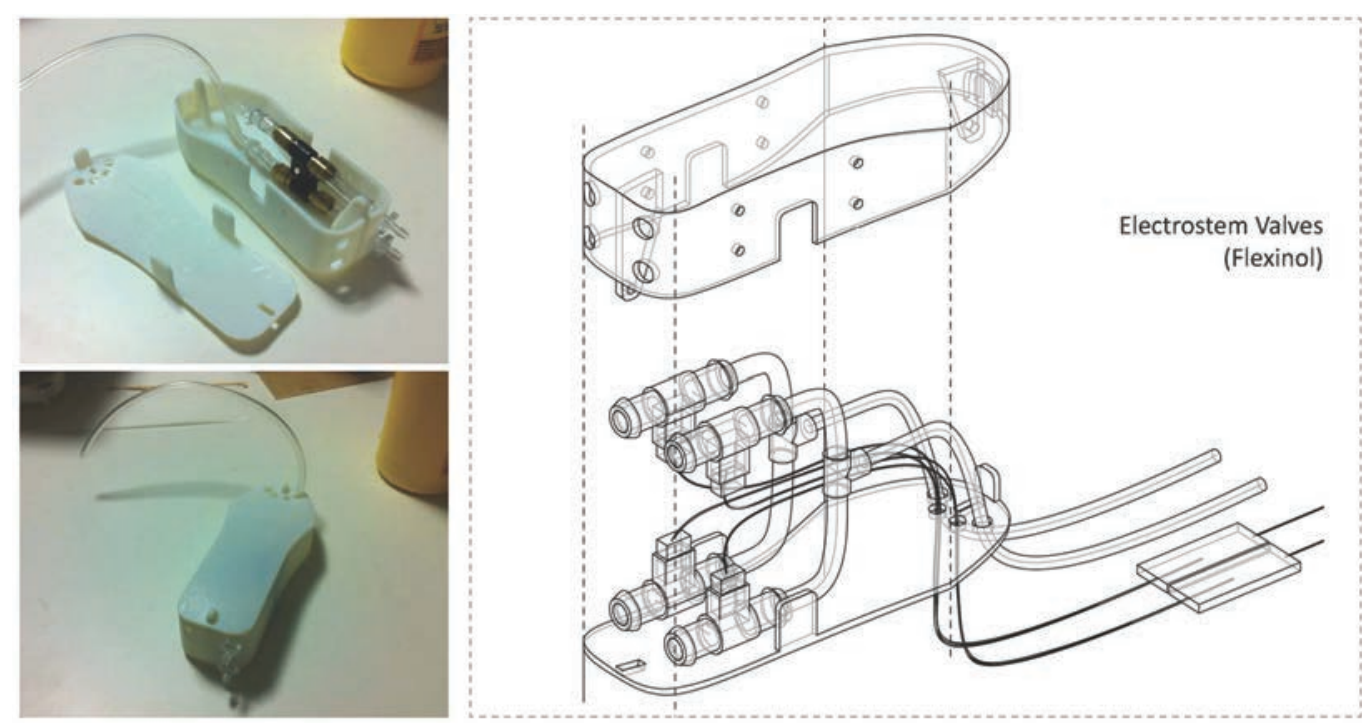

Figure 23:

Chassis configuration.

\subsection{Potential applications}

Flexibility and versatility enables the SMoPS to be used in a wide range of architectural applications, including the building envelope, interior wall, floor, 
and ceiling. The key advantages of utilizing the SMoPS as building components and systems is the ability to transform what were commonly considered static and passive elements into dynamic and active (responsive) elements. The sensor, actuation, and control methods can be creatively combined and configured to create various types of interactive and/or responsive systems and environments. Individual module shapes can adapt to project-specific needs. The Public Library of the City of Cambridge (MA) was chosen as a site to virtually implement various potential applications of the SMoPS. 3D animation techniques were utilized to effectively visualize the dynamic properties of various applications.

The first application is an exterior facade system that can dynamically regulate its opacity. The diagonal grid screen is created using soft diamond shaped modules. Each module has a transparent chamber embedded in the slot that runs vertically from the top point to the bottom point of the diamond shape. When the transparent chamber expands, the slot is pushed open. A simple photo sensor or a UV sensor can be embedded in each module to respond to the brightness or the UV light from the sun. In addition, the occupants can also control the modules in order to look outside or regulate the natural lighting locally.

Figure 24:

Responsive facade animation sequence.
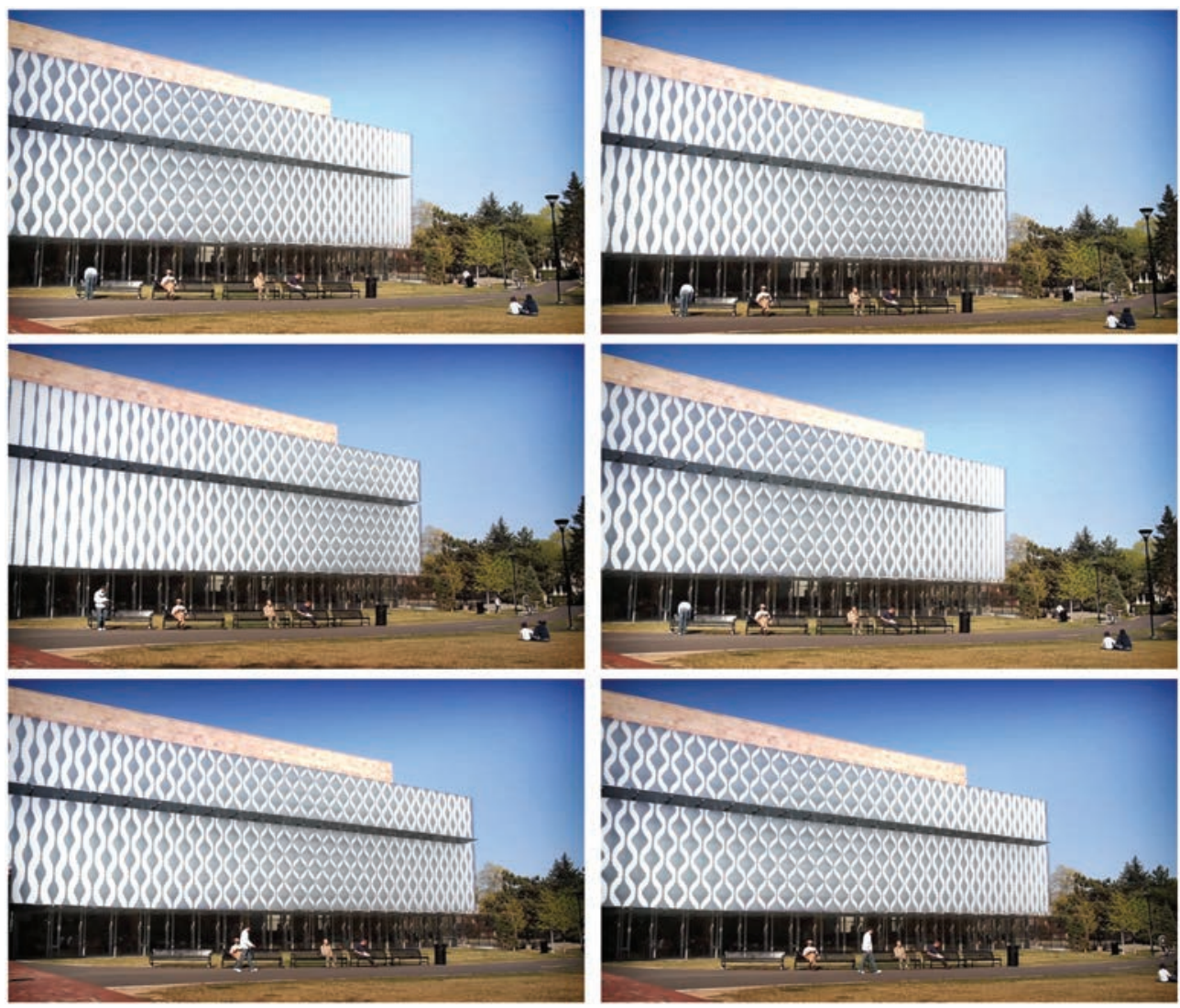

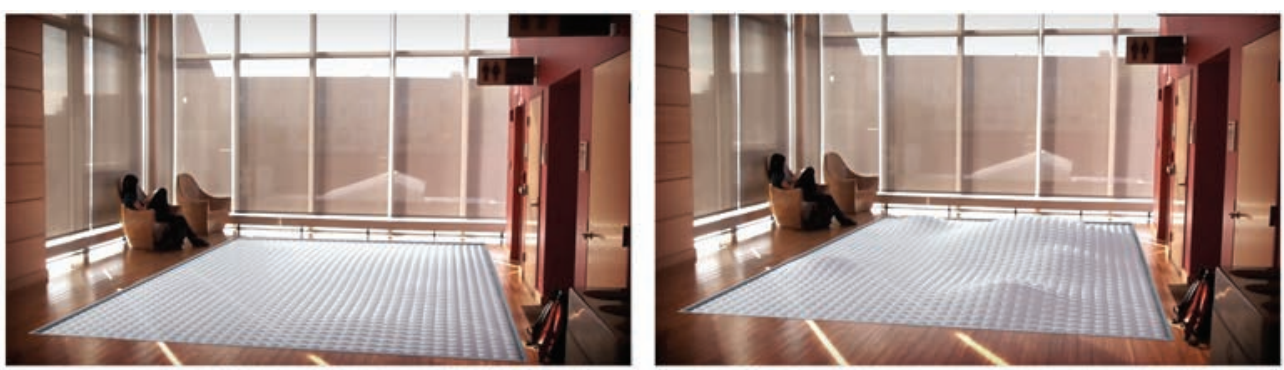

Figure 25:

Animation sequence of responsive floor
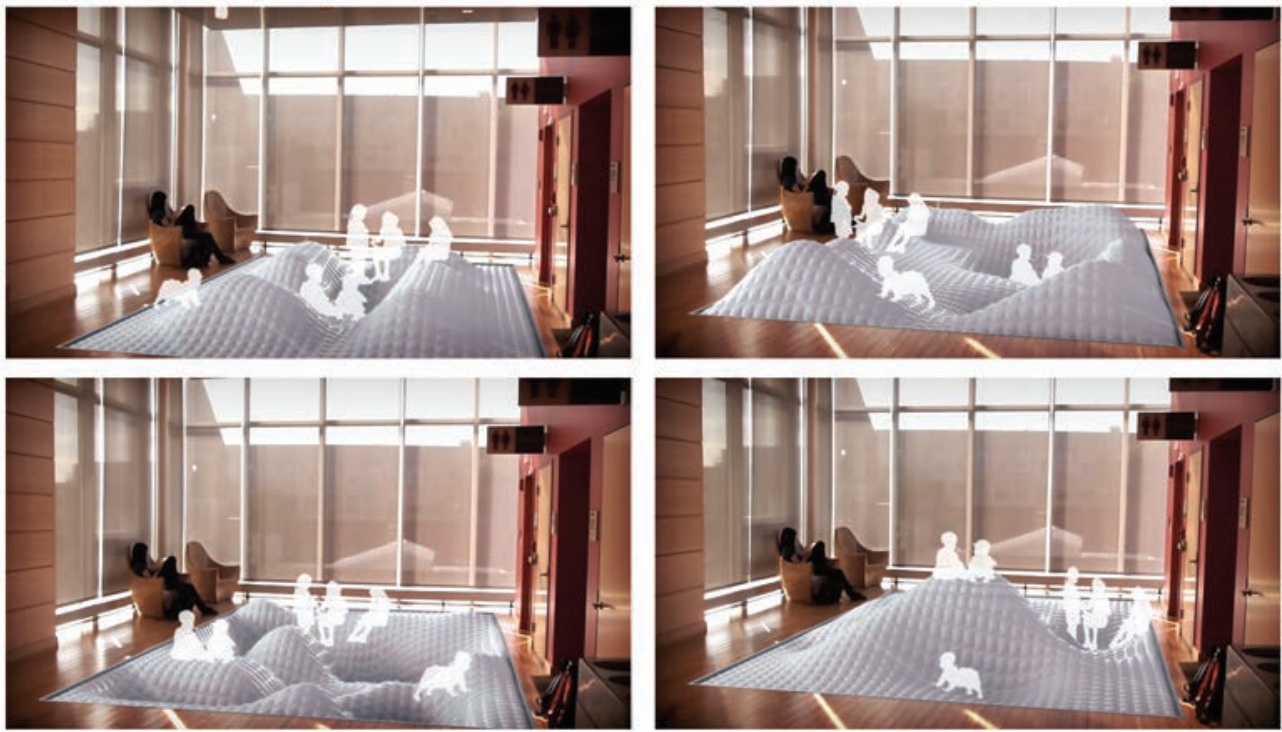

The second application was envisioned to be a dynamic floor that can function as an interactive playground for the children's area. Each kinetic module is embedded within a soft surface material, expanding and extending and thereby changing the surface topography. The softness of the overall system makes it a safe for children to play on. Pressure sensors or capacitive sensors can be utilized to make the system responsive to the weight or to the proximity of children. Several activity-specific configurations (e.g. book reading session, sing along session, etc.) could be pre-programmed into the microcontroller.

The third potential application is a dynamic ceiling system that responds to the proximity of the occupants. Capacitive sensors can be embedded into the modules, and by creating a capacitive sensor network, thus transforming the entire ceiling into an interactive surface that registers precise location data. A lighting system is proposed such that embedded LED lights gradually up and create a personal spot lights as the modules extend towards the occupant. 
Figure 26:

Animation sequence of responsive ceiling
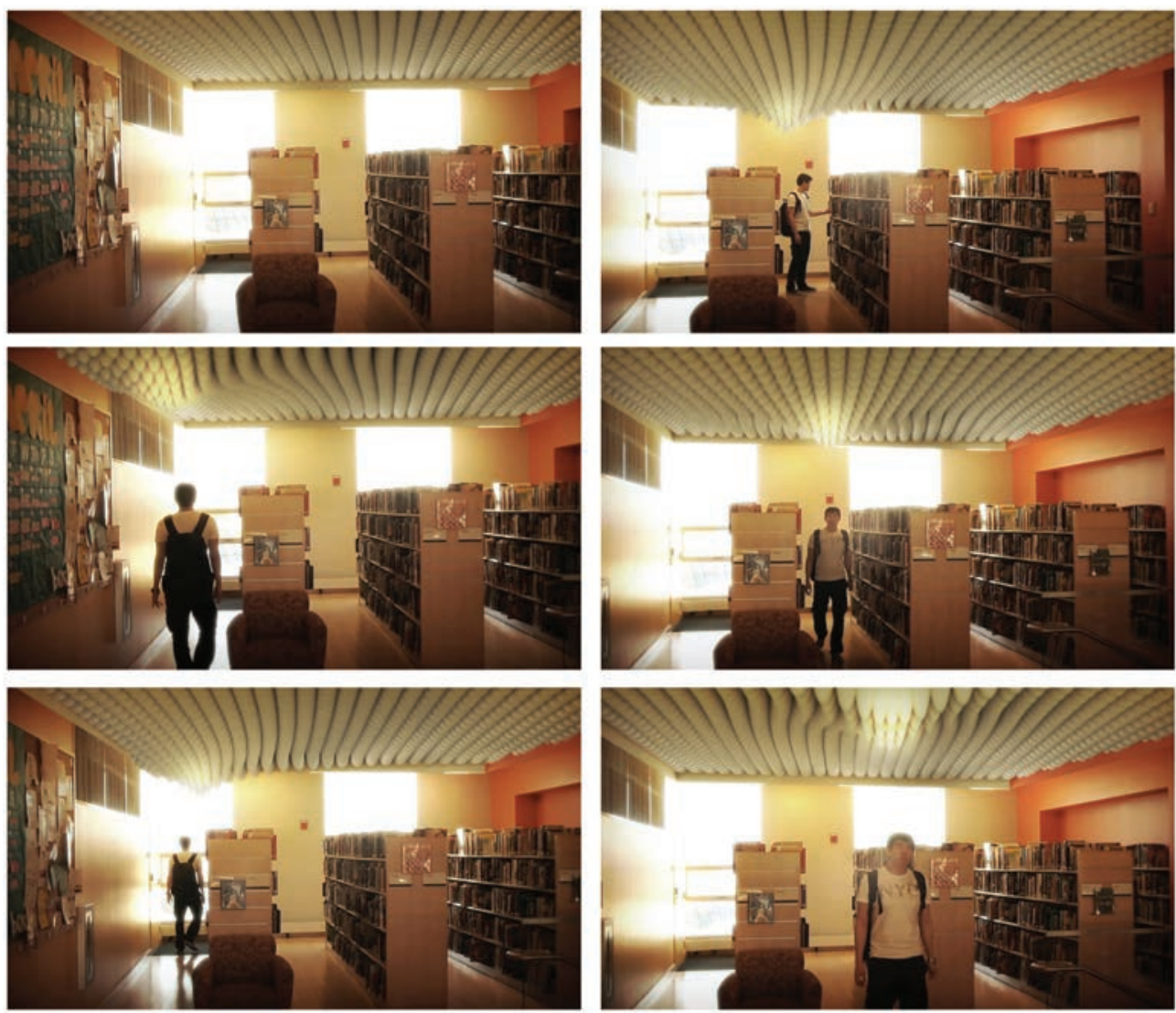

The final application is an interior shading surface that responds to the presence of nearby occupants. This application is similar to the $3 \mathrm{rd}$ stage prototype of the SMoPS experiment using conductive gel for creating a capacitive sensor. However, in order to make the surface as thin as possible, only the bending motion is utilized and the expansion and extension motions were excluded. Each module is essentially a thin box with channels running diagonally. 4 modules that consist of modules mirrored around a point forms a set within the field. When pneumatic pressure is applied, each set is bent into a diamond shaped opening.

\section{ANALYSIS AND DISCUSSION}

The key goal of BISBS research is to investigate the means and methods of creating dynamic and active building systems that echo living biological systems. In order for BISBS to become a versatile yet robust system, it needs to bring together various concepts and technologies from biologically-inspired smart technology. BISBS is conceived to be the technological infrastructure that allows a building to regulate itself as well as to interact with the occupants, much like living organisms maintain 

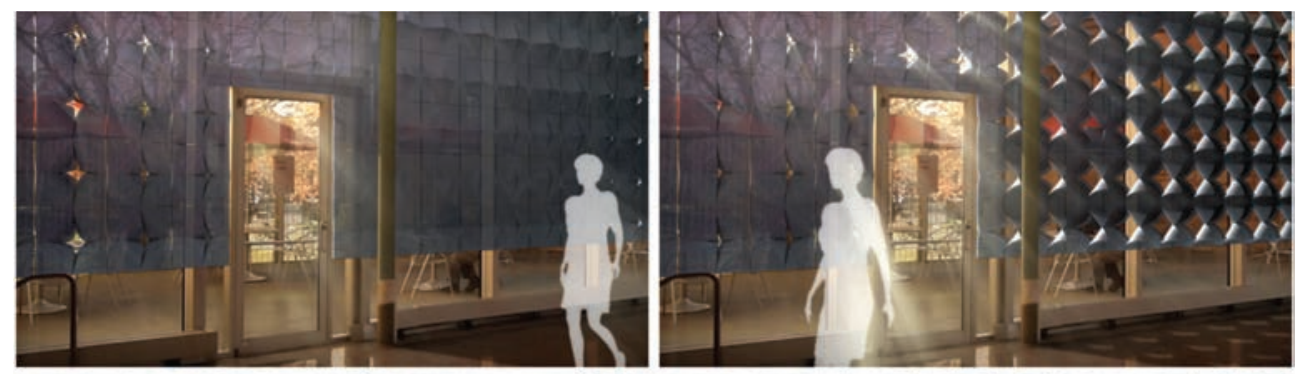

Figure 27: Animation sequence of responsive screen.
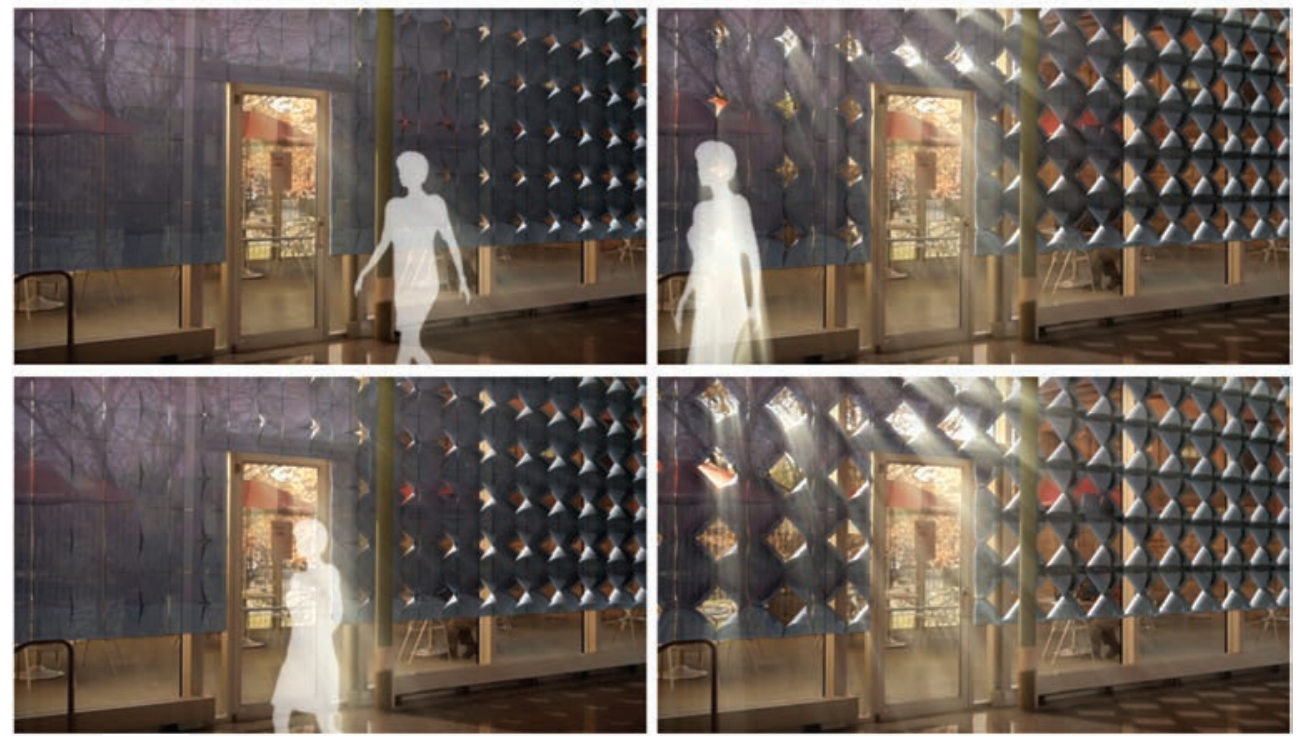

homeostasis and respond to external stimuli.

Composition and integration of various technological developments in material, sensor, actuation, and control are essential for developing BISBS. The biological inspiration is integrated into all aspects of the system, including the concept, physical manifestation, and technology. The main challenge is to combine flexibility and system robustness, and optimize the system for fabrication and performance. Despite its technical complexity the goal was to maintain visual elegance and beauty. Additional requirements include the ability to maintain, repair, and replaced modules easily; versatility to adapt to the module design to various design intentions and functional needs; ability to scale up and down according to a project's requirements; and interchangeability of sensors, actuators, and control methods.

The robustness and flexibility of BISBS is achieved through modular design and hierarchical assembly logic. On the one hand, the reconfigurablity and replacability of BISBS modules in order to adapt to different functional, spatial, or maintenance requirements are the key strategy of the system for achieving robustness. As seen in the SMoPS design experiment, the system consists of individual modules that are integrated assemblies of the material 
body, sensor, actuation and control components. Each module is a standalone building block that can be mass-produced and assembled into any type of configuration and size. Also, since each module is essentially autonomous with a complete physical computing loop, each of them can be individually tested, fixed, reconfigured, or replaced when necessary.

On the other hand, the hierarchical assembly logic contributes to the flexibility of the system. The outer skin is the main interface; the inner chassis is the casing for physical computing components; and the power supplier (tubes or wires) is the connector as well as the structure of the modules. The outer skin can be designed in any shape or size depending on the functional requirements or ornamental purposes, and the physical components (sensor, actuation, and control) within the chassis can be configured in various ways. Since the power supplier functions both as connector and structure, the entire system can scale up and down without creating a secondary substructure.

Until now, the role of designers and architects has been focused on participating in the ideation and visualization of building systems, while technological research and development were essentially led by scientists and engineers. It was stated earlier that the increased accessibility and availability of design tools and prototyping methods is now enabling architects to directly experiment and develop new technologies and material systems. The design and development of SMoPS has taken full advantage of this change. The various research and technological developments could be compartmentalized and quickly tested through prototyping. Simulation also had a major role during the development process. Instead of fabricating multiple units, which require significant time and expense, open source software was utilized to bridge the digital representation of the field of modules with the physical computing components. Different control strategies using a range of physical components could be tested quickly using this method. Computational simulation enabled real time analysis and verification of them.

\section{CONCLUSION}

BISBSs enable buildings to acquire the adaptive and versatile attributes of living systems. The design studies showed that BISBSs can be effectively developed and implemented in the built environment. Within the biological paradigm in contemporary architecture the performative aspects of material properties are tightly related to recent developments in smart control technologies. Architects and designers now operated in a newly expanded scale ranging from nano/micro scale to building scale, influencing the functionality of the materials and modes of building adaptation through an expanded set of strategies and techniques. Studies of growth, behavior, and dynamic optimization [14] are instrumental when pursuing design based on biological precedents. Smart technology is a broad field of study that not 
only includes smart materials at various scales but also components, assemblies and systems. It can provide architects with the tools and methods essential for designing smart buildings that can effectively adapt to the ever-evolving social, cultural, and environmental changes.

As the research and developments in molecular technology including material science, biotechnology and nanotechnology [15] further matures, more and more advanced material systems with adaptive and smart attributes will be available to architects and designers. Comparable to the effect of increased availability of wood, masonry, and glass in the postindustrialization era [3], which enabled architects to experiment with various performative aspects such as transparency, light, and heat; the increased availability of smart materials in the near future is likely to further expand design scope. This paper shows strategies how architects can conduct creative experiments with dynamic adaptation towards enhanced building performance.

The exponential growth of biologically inspired approaches in science, engineering, and design is increasingly faced with the challenge of coordinating among multi-disciplinary fields of studies and industries. To master the challenge of this expanded mode of multi-disciplinary work designers need to embrace scientific modes of research, and scientists and engineers need to accept that architects and designers pursue synthetic modes of work that lead to new outcomes. Designers and architects, trained to be generalists, can effectively take on the coordination role between various entities and fields, looking at the broader picture with an open and flexible mind. These newly formed collaborative endeavors will fully enable society to critically pursue biological paradigms in design, and unleash the potentials of smart technology for a better future.

As stated in the second chapter, BISBS is the intersection between biologically-inspired design, smart technology and building systems. The key criteria for the evaluation of BISBS is how rigorous these three fields are simultaneously considered and how successfully it is manifested as an integrated system. Although SMoPS has been developed as a design experiment, it is only one limited example among the various possibilities of BISBS. Therefore, in order to fully understand and take advantage of the unlimited potentials of BISBS, more systems utilizing the approaches, criteria and guidelines established by this paper should be designed, developed and tested.

\section{ACKNOWLEDGEMENTS}

The authors would like to thank professors Takehiko Nagakura, Leah Buechley and Allen Sayegh for the comments and insights throughout the research. We would also like to thank Woong Ki Sung and Namju Lee for dedicating their time to the research during the prototyping stage and visualization stage. 


\section{REFERENCES}

I. Kirkland, D., A Process-oriented Architecture, in: Brookes, Alan and Poole, Dominique, eds., Innovation in Architecture. Spon Press, London; New York, 2003, 4I-54.

2. Banham, R.. The Architecture of the Well-Tempered Environment. 2nd Ed. University of Chicago Press, Chicago, 1984.

3. Elliott, C. D., Technics and architecture: the development of materials and systems for buildings. MIT Press, Cambridge, 1992.

4. http://www.kmccontrols.com/products/Understanding_Building_Automation_ and_Control_Systems.aspx [10-6-20|3].

5. Dieter, N.,Adaptronics:A concept for the development of adaptive and multifunctional structures, in: Hartmut, J., ed., Adaptronics and smart structures, Springer, Berlin, 2007, I-8.

6. Addington, M. and Schodek, D., Smart Materials and Technologies: For the Architecture and Design Professions. Routledge, 2004.

7. Wadhawan, V. K., Smart structures : Blurring the distinction between the living and the nonliving, Oxford University Press, New York, 2007.

8. http://www.philipbeesleyarchitect.com [10-17-2013].

9. Bachman, L. R., Integrated Buildings: the systems basis of architecture, John Wiley \& Sons, Inc., Hoboken, NJ, 2003.

10. Kier,W. M., and Smith, K. K., Tongues, tentacles and trunks: the biomechanics of movement in muscular-hydrostats, Zoological Journal of the Linnean Society, 1985, $83,307-32$

I I. Ilievski, F., Mazzeo, A. D., Shepherd R. F., Chen, X and Whitesides, G. M., Soft Robotics for Chemists, Angewandte Chemie, 20I I, 123, 1930-1935.

12. Baxter, L. K., Capacitive Sensors, John Wiley and Sons, New York, 1996.

13. http://fireflyexperiments.com [10-17-2013].

14. Fox, M. and Kemp, M., Interactive Architecture. Princeton Architectural Press, New York, 2009.

15. Meyer, C. and Davis, S., It's Alive:The Coming Convergence of Information, Biology, and Business. Crown Publishing Group. Kindle Edition, 2003.

\section{Daekwon Park and Martin Bechthold}

Harvard University

Graduate School of Design

48 Quincy Street, Gund Hall, Cambridge, MA 02138

dparkI,mbechthold@gsd.harvard.edu 
